\title{
Earth Observing System Covariance Realism
}

\author{
Waqar H. Zaidi ${ }^{1}$ and Matthew D. Hejduk ${ }^{2}$
}

\begin{abstract}
The purpose of covariance realism is to properly size a primary object's covariance in order to add validity to the calculation of the probability of collision. The covariance realism technique in this paper consists of three parts: collection/calculation of definitive state estimates through orbit determination, calculation of covariance realism test statistics at each covariance propagation point, and proper assessment of those test statistics. An empirical cumulative distribution function (ECDF) Goodness-of-Fit (GOF) method is employed to determine if a covariance is properly sized by comparing the empirical distribution of Mahalanobis distance calculations to the hypothesized parent 3-DoF chisquared distribution. To realistically size a covariance for collision probability calculations, this study uses a state noise compensation algorithm that adds process noise to the definitive epoch covariance to account for uncertainty in the force model. Process noise is added until the GOF tests pass a group significance level threshold. The results of this study indicate that when outliers attributed to persistently high or extreme levels of solar activity are removed, the aforementioned covariance realism compensation method produces a tuned covariance with up to 80 to $90 \%$ of the covariance propagation timespan passing (against a $60 \%$ minimum passing threshold) the GOF tests-a quite satisfactory and useful result.
\end{abstract}

\section{Nomenclature}

$\begin{array}{ll}B C & =\text { Ballistic Coefficient } \\ C A M & =\text { Command Authorization Meeting } \\ C A R A & =\text { Conjunction Assessment Risk Analysis } \\ C C S D S & =\text { Consultative Committee for Space Data Systems } \\ C D F & =\text { Cumulative Distribution Function } \\ C M E & =\text { Coronal Mass Ejection } \\ D o F & =\text { Degrees-of-Freedom } \\ E C I & =\text { Earth-Centered-Inertial } \\ E D F & =\text { Empirical Distribution Function } \\ E C D F & =\text { Empirical Cumulative Distribution Function } \\ E O S & =\text { Earth Observation System } \\ E S M O & =\text { Earth Science Mission Operations } \\ F D S & =\text { Flight Dynamics System } \\ G N & =\text { Ground Network } \\ G S F C & =\text { Goddard Space Flight Center } \\ G O F & =\text { Goodness-of-Fit } \\ J S p O C & =\text { Joint Space Operations Center } \\ N A S A & =\text { National Aeronautics and Space Administration } \\ N O A A & =\text { National Oceanic and Atmospheric Administration } \\ O D & =\text { Orbit Determination } \\ O E M & =\text { Orbit Ephemeris Message } \\ O / O & =\text { Owner/Operator } \\ R I C & =\text { Radial, In-Track, Cross-Track } \\ R M M & =\text { Risk Mitigation Maneuver } \\ & \end{array}$

${ }^{1}$ Technical Lead, Earth Observing System Flight Dynamics System, Omitron, Inc., 7051 Muirkirk Meadows Drive, Suite A, Beltsville, MD 20705, junior member AIAA.

${ }^{2}$ Chief Engineer, NASA Robotic CARA, Astrorum Consulting LLC, 10006 Willow Bend Drive, Woodway TX, 76712, senior member AIAA. 


$$
\begin{array}{ll}
R M S & =\text { Root-Mean-Square } \\
P_{C} & =\text { Probability of Collision } \\
p \text {-value } & =\text { Probability Value } \\
T C A & =\text { Time of Closest Approach } \\
T D R S & =\text { Tracking Data Relay Satellite } \\
T D R S S & =\text { Tracking Data Relay Satellite System } \\
S N & =\text { Space Network } \\
S T K & =\text { Satellite Tool Kit }
\end{array}
$$




\section{Introduction}

$\mathrm{T}$ he NASA Aqua and Aura Earth Observation System (EOS) missions have adopted a covariance realism assessment and compensation method that ensures their predicted covariances represent realistic distributions of their trajectory propagation errors. The assessment process identifies the quality of the covariances, whereas the compensation or tuning process works to improve it. Each EOS mission trajectory is propagated daily from an updated definitive state estimate and predicted atmospheric model. The covariances corresponding to the propagated trajectories are constructed using linearized covariance propagation. The definitive state estimate and its covariances are determined daily by passing orbital observations through an extended Kalman filter. In fact, the covariance realism assessment and compensation method uses inferential statistics in which behavioral conclusions for a large population (represented by covariances propagated for a fixed interval over a long period of time) are drawn using sample data (represented by a subset of the propagated covariances).

The calculation of the Probability of Collision $\left(\mathrm{P}_{\mathrm{C}}\right)$ for two objects in conjunction requires two key measures: (1) the miss distance or difference between the predicted state estimates of the two conjuncting objects and (2) the two objects' covariances at the Time of Closest Approach (TCA). Figure 1 depicts two objects in conjunction. R is the radial axis that "points from the Earth's center along the radius vector toward the centroid of the primary object", I as the in-track axis that "points in the direction of (but not necessarily parallel to) the velocity vector", and C as the cross-track axis normal to the orbital plane. ${ }^{1}$ The encounter coordinate system is defined with origin $o$ at the centroid of the primary object. The " $z$-axis points in the direction of the relative velocity vector $\Delta v$, the $x$-axis points in the direction of closest approach vector between the two trajectories (i.e., the line of intersection of orbital plane; it is in the radial direction when two orbits are circular)." "The conjunction plane is defined to be perpendicular to the relative velocity vector between the two conjuncting objects. The relative position vector $\Delta \boldsymbol{r}$ and the $y$-axis, completing the right-hand system, lie in this plane. The approach angle, $\varphi$, is the angle between the two velocity vectors, assuming a relatively minor radial separation at the Time of Closest Approach (TCA) of the two conjuncting objects.

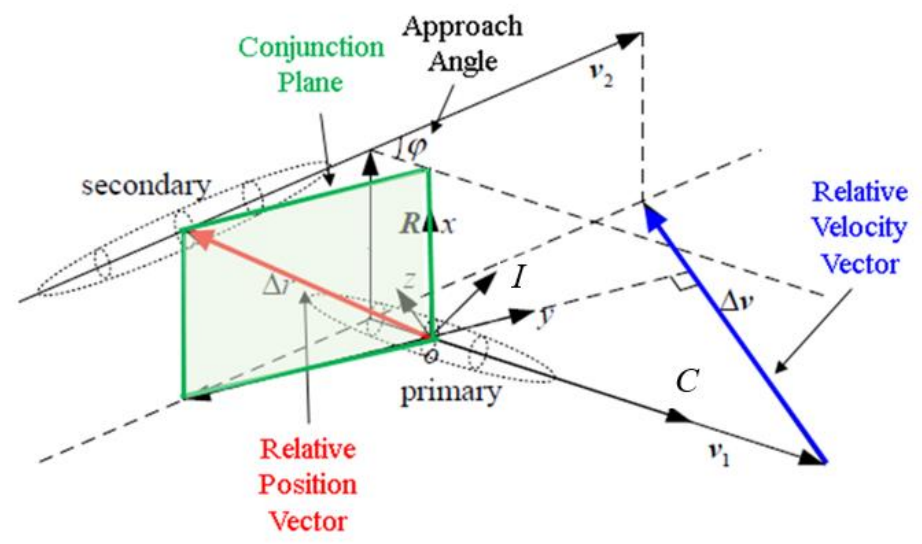

Figure 1. Conjunction geometry. ${ }^{1}$

For most conjunctions, the calculation of the $\mathrm{P}_{\mathrm{C}}$ is performed by projecting the combined covariances of the primary and secondary objects onto the conjunction plane. As a result, for two objects in conjunction with a low miss distance and equally sized covariances, a realistic representation of the primary object or Owner/Operator $(\mathrm{O} / \mathrm{O})$ covariance is critical to producing a realistic Pc and, thus, performing conjunction risk analysis. This study describes the techniques developed for the EOS missions to produce realistic O/O covariances.

This paper is divided into four main sections. The first section provides a brief description of the orbit determination and trajectory propagation processes for both EOS spacecraft that produce their definitive state estimates and covariances as well as their predictive state estimates and covariances. The next section describes the adopted covariance realism assessment and compensation method. The third section describes outlier trajectory identification - a process vital to covariance tuning. The essence of this paper lies in its fourth section, which presents a covariance realism assessment and compensation example for Aqua covariances from August to November 2014. This section compiles Aqua predicted covariances and trajectory propagation errors, performs the assessment of the covariances, identifies outlier propagations, and proceeds to the tuning of the set of covariances. 
The result is a set of covariances exhibiting high levels of covariance realism. The fourth and final describes the automated covariance assessment algorithm developed by the EOS FDS team. Specifically, the algorithm is able to automatically perform daily distribution testing, automatically determine the presence of outlier trajectory propagations, and notify the analyst when tuning of the covariance is required. This section demonstrates how to maintain an operational spacecraft's tuned covariance for periods devoid of persistently high or extreme solar activity.

\section{Definitive and Predicted State Estimates and Errors}

The NASA Goddard Space Flight Center (GSFC) Flight Dynamics Facility (FDF) provides the definitive Orbit Determination (OD) capability for the Aqua and Aura spacecraft. Direct tracking of the EOS missions consists of Tracking and Data Relay Satellite(TDRS)/Space Network (SN) two-way range and Doppler tracking and Ground Network (GN) Doppler tracking. These measurements are ingested by the Orbit Determination Tool Kit (ODTK®) software developed by Analytical Graphics, Inc., which simultaneously estimates the TDRS and EOS mission orbits. ODTK employs a Kalman filter measurement update, but the time-update equations are non-Kalman, meaning the software uses process-noise based on the uncertainty in the physical models to enable the calculation and propagation of a more realistic definitive covariance. ${ }^{2}$ Furthermore, ODTK uses a fixed-interval smoother that combines filtered state estimates and their errors in reverse chronological order. In fact, the smoothing application compensates for discontinuities caused by state corrections to produce the definitive orbit. The Aqua and Aura spacecraft propagation settings in ODTK are listed in Table 1.

Table 1. ODTK Propagation Settings for Aqua and Aura.

\begin{tabular}{|l|c|}
\hline \multicolumn{1}{|c|}{ Property } & Value \\
\hline \hline Gravity Model & EGM 96 \\
\hline Gravity Degree/Order & 50 \\
\hline Solid Tides & On \\
\hline Time Dependent Tides & On \\
\hline Third Bodies & Sun and Moon \\
Density Model & Jacchia-Roberts \\
\hline Coefficient of Drag, $C_{D}$ & Based on Daily OD \\
\hline Area $\left(\mathrm{m}^{2}\right)$ & 47.95 \\
\hline Solar Radiation Pressure Coefficient, $\mathrm{C}_{\mathrm{R}}$ & 1.7 \\
\hline Integration Method & RKF 7(8) \\
\hline Use of VOP & On \\
\hline Error Tolerance & $1.00 \mathrm{E}-13$ \\
\hline
\end{tabular}

Each operational day, $24 \mathrm{hrs}$ of TDRS and GN measurements are run forward through the filter and then the EOS mission orbit is smoothed backward using 2.5 days worth of measurements. As a result, ODTK generates "filtered" and "smoothed" OD solutions in addition to estimating a relative correction to the Ballistic Coefficient (BC). The smoothed OD ephemeris and $\mathrm{BC}$ correction value are delivered to the FDS team in preparation for the predicted state estimate and covariance propagation.

The EOS Flight Dynamics System (FDS) team propagates a 12:00 UTC definitive state estimate and covariance for seven days using the commercial-off-the-shelf software product FreeFlyer® developed by a.i. solutions, Inc.. The result is sent to the NASA Robotic Conjunction Assessment and Risk Analysis (CARA) team, which in turn uses resources from the Joint Space Operations Center (JSpOC) to estimate the miss distance of any objects that are predicted to be in conjunction with the Aqua or Aura spacecraft. The CARA team is responsible for computing $\mathrm{P}_{\mathrm{C}}$ using the conjunction information provided by the JSpOC and the tuned $\mathrm{O} / \mathrm{O}$ covariance supplied by the EOS FDS team.

The EOS FDS team propagates the Aqua and Aura definitive covariances using linear mapping via the following equation:

$$
P(t)=\varphi P\left(t_{0}\right) \varphi^{T}+\Gamma Q(t) \Gamma^{T}
$$


Note that $\varphi$ is the State Transition Matrix, $\Gamma$ is the Process Noise Transition Matrix, $Q$ is the Process Noise Matrix, $P\left(t_{0}\right)$ is the definitive state estimate covariance at $t_{0}$, and $P(t)^{*}$ is the predicted state estimate covariance at time $t^{3}$

The method of adding process noise to the propagation of the definitive covariance is known as state noise compensation (SNC). In this method the process noise is compensating for the trajectory propagation errors in the linearized dynamics of the force model, other truncations (such as a reduced geopotential fidelity), and other unmodeled forces. These trajectory propagation errors can be represented by the component acceleration variances specified in an acceleration error vector. An example of the radial predicted covariance with and without process noise addition appears in Figure 2.
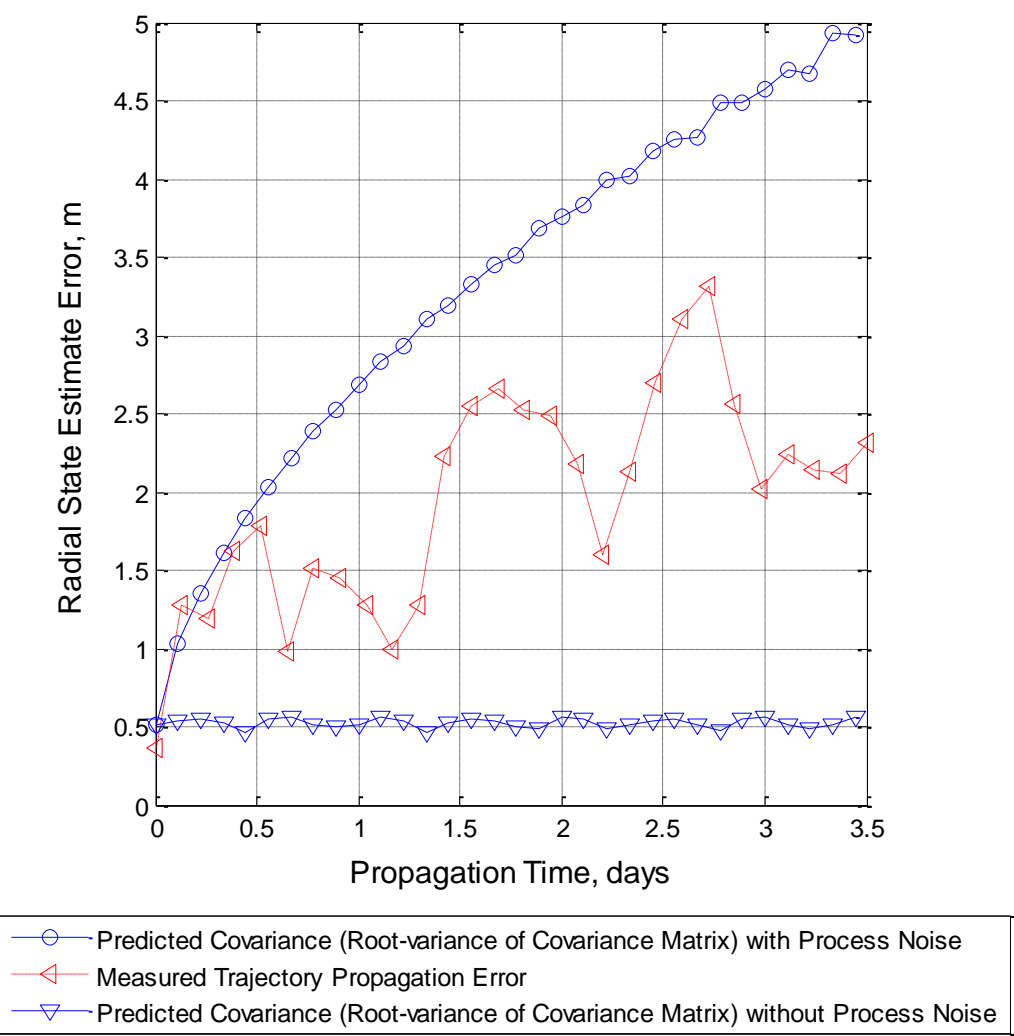

Figure 2. State Noise Compensation example for a radial predicted covariance (equivalent to the radial rootvariance of the covariance matrix) and measured trajectory propagation error. Without the application of process noise, the propagation error is misrepresented by the covariance.

It is evident that without state noise compensation the predicted trajectory propagation error is severely mischaracterized. Therefore, an $\mathrm{O} / \mathrm{O}$ covariance that is not tuned will not provide a reasonable representation of the trajectory propagation error, which will in turn affect the calculated $\mathrm{P}_{\mathrm{C}}$ and thus impact the fidelity of the conjunction risk analysis. Accordingly, proper tuning of the covariance is controlled by integrating random acceleration uncertainties into the propagation of the covariance using Eq. (1) where the process noise matrix, $Q(t)$, is built using acceleration variances through the technique demonstrated in Ref. 3. The governing equations are given below in Eqs. (2) through (4) for reference.

$$
Q(t)=\left[\begin{array}{cc}
Q_{\mathrm{RIC}} & 0_{1 \times 6} \\
0_{6 \times 1} & Q_{\mathrm{C}_{\mathrm{D}}}
\end{array}\right],
$$

\footnotetext{
* Although the correlation coefficients of a covariance matrix are known as its co-variances, this paper uses the common notion in which the standard deviations or root-variances of the covariance matrix (the square roots of its diagonal terms) are referred to as its component covariances.
}

American Institute of Aeronautics and Astronautics 
where

$$
Q_{\mathrm{RIC}}=\left[\begin{array}{ll}
\frac{\Delta T^{4}}{3} I \cdot \bar{q}_{a c c} & \frac{\Delta T^{3}}{2} I \cdot \bar{q}_{a c c} \\
\frac{\Delta T^{3}}{2} I \cdot \bar{q}_{a c c} & \Delta T^{2} I \cdot \bar{q}_{a c c}
\end{array}\right]
$$

and

$$
\bar{q}_{a c c}=\left[\begin{array}{c}
\sigma_{\ddot{\mathrm{R}}} \\
\sigma_{\ddot{\mathrm{I}}} \\
\sigma_{\ddot{\mathrm{C}}}
\end{array}\right]^{2} \text { and } I=\left[\begin{array}{ccc}
1 & 0 & 0 \\
0 & 1 & 0 \\
0 & 0 & 1
\end{array}\right]
$$

Note that $Q_{\mathrm{C}_{\mathrm{D}}}$ is the variance in the drag coefficient, $\mathrm{C}_{\mathrm{D}}, \Delta T$ is the Propagation Time Step, and $\sigma_{i}$ represents each component acceleration variance.

Consequently, the acceleration variances manipulate the uncertainty in the position and velocity components of the predicted covariance. The $\mathrm{BC}$ correction received from the FDF is converted to a $\mathrm{C}_{\mathrm{D}}$ correction and attached to the definitive epoch covariance as the seventh diagonal term of the matrix. The EOS FDS team uses slightly different propagation settings in its software from those used in ODTK by the FDF in Table 1. The EOS FDS values are listed in Table 2. Attempts to closely match the propagations are underway; it remains to be seen if the usage of two separate software for the EOS trajectory estimation and propagation is impacting the covariance assessment and compensation method in this paper.

Table 2. FreeFlyer Propagation Settings for Aqua and Aura.

\begin{tabular}{|l|c|}
\hline \multicolumn{1}{|c|}{ Property } & Value \\
\hline \hline Gravity Model & JGM2 \\
\hline Gravity Degree/Order & 30 \\
\hline Solid Tides & Off \\
\hline Time Dependent Tides & Off \\
\hline Third Bodies & Sun and Moon \\
\hline Density Model & Jacchia-Roberts \\
\hline Coefficient of Drag, $C_{D}$ & Based on Daily OD \\
Area $\left(m^{2}\right)$ & 47.95 \\
\hline $\begin{array}{l}\text { Solar Radiation Pressure } \\
\text { Coefficient, } C_{R}\end{array}$ & 1.4 \\
\hline Integration Method & RKF $8(9)$ \\
\hline Use of VOP & Off \\
\hline Error Tolerance & $1.00 \mathrm{E}-9$ \\
\hline
\end{tabular}

\section{Covariance Realism Assessment and Compensation Method}

The Mahalanobis distance of a covariance at a particular propagation point represents the ratio of the predicted trajectory propagation error to the covariance's prediction. For a trivariate distribution, the Mahalanobis distance has an expected value of 3. A group of the squares of such calculations should conform to a chi-squared distribution with 3-DoF. ${ }^{4}$ The chi-squared statistic is computed using the vector of predicted trajectory propagation error estimates, $\varepsilon$, and the predicted covariance matrix, $P$; that is,

$$
\chi_{3 \mathrm{DoF}}^{2}=\varepsilon P^{-1} \varepsilon^{T} \approx\left(\frac{\varepsilon_{R}}{\sigma_{R}}\right)^{2}+\left(\frac{\varepsilon_{I}}{\sigma_{I}}\right)^{2}+\left(\frac{\varepsilon_{C}}{\sigma_{C}}\right)^{2}
$$


The approximation on the right side holds when correlation terms are small and can be neglected; it gives an intuitive sense of why the square of the Mahalanobis distance $\left(\varepsilon P^{-1} \varepsilon^{T}\right)$ is equal to the sums of the squares of random normal deviates, with the number of degrees of freedom equal to the number of such random variables summed.

If the propagated trajectory errors are accurately described by the propagated covariance at each propagation point, the set of ratios of the errors to the covariances' expectations, calculated as Mahalanobis distances, should conform to a chi-squared distribution with 3 Degrees of Freedom (DoF). ${ }^{4}$ A Goodness-of-Fit (GOF) test is performed to assess how well the empirical distribution of Mahalanobis distances for each group of trajectories conforms to the expected 3-DoF chi-squared hypothesized parent distribution. Finally, the Rosner Outlier Identification test is performed to investigate the existence of outlier trajectory propagations, ones that can skew the distribution and produce an unrealistic covariance. ${ }^{5,6}$

The assessment of the realism is confined to the position portion of the covariance, as only the position portion of the covariance is used in conjunction assessment (CA) processes for EOS missions. Furthermore, the assessment and compensation method employs an empirical cumulative distribution function (ECDF) GOF technique drawing from quadratic, rather than supremum statistics. This technique evaluates how well an empirical distribution conforms to a hypothesized parent distribution by examining the summation of a function of the squares of the deviations between the two distributions rather than examining merely the largest single deviation. The ECDF test statistic is given below:

$$
Q=n \int_{-\infty}^{\infty}\left[F_{n}(x)-F(x)\right]^{2} \psi(x) d x
$$

Note that $Q$ is the ECDF Test Statistic, $n$ is the number of empirical samples, $F_{n}(x)$ is the Empirical CDF, $F(x)$ is the Hypothesized Parent CDF and $\psi(x)$ is the Weighting Function.

Examples of quadratic GOF test statistics include the Cramér-von Mises, Watson, and Anderson-Darling statistics. This analysis exploits the more permissive Cramér-von Mises statistic, or " $Q$-statistic," due to the likelihood of outliers; it has been observed that this more robust approach is better suited to the observed outlier characteristics of the Aqua predicted ephemeris generation process. The Cramér-von Mises $Q$-statistic sets $\psi$ to unity, thus weighting data across the entire distribution equally instead of weighting data in the tails of the distribution more heavily. A $p$-value (probability value) is obtained for each $Q$-statistic in Eq. (6) using a published table of $p$-values built using Monte Carlo simulations. ${ }^{7}$ A $p$-value provides the analyst with the likelihood the empirical distribution is drawn from its hypothesized parent distribution. Furthermore, a confidence interval or $p$ value threshold that indicates a "pass" or "fail" is set as a passing criteria; this analysis uses a confidence interval of $2 \%$ or higher, a result often accepted in GOF practice. Figure 3 illustrates the deviation between an empirical and a hypothesized parent 3-DoF chi-squared distribution. 


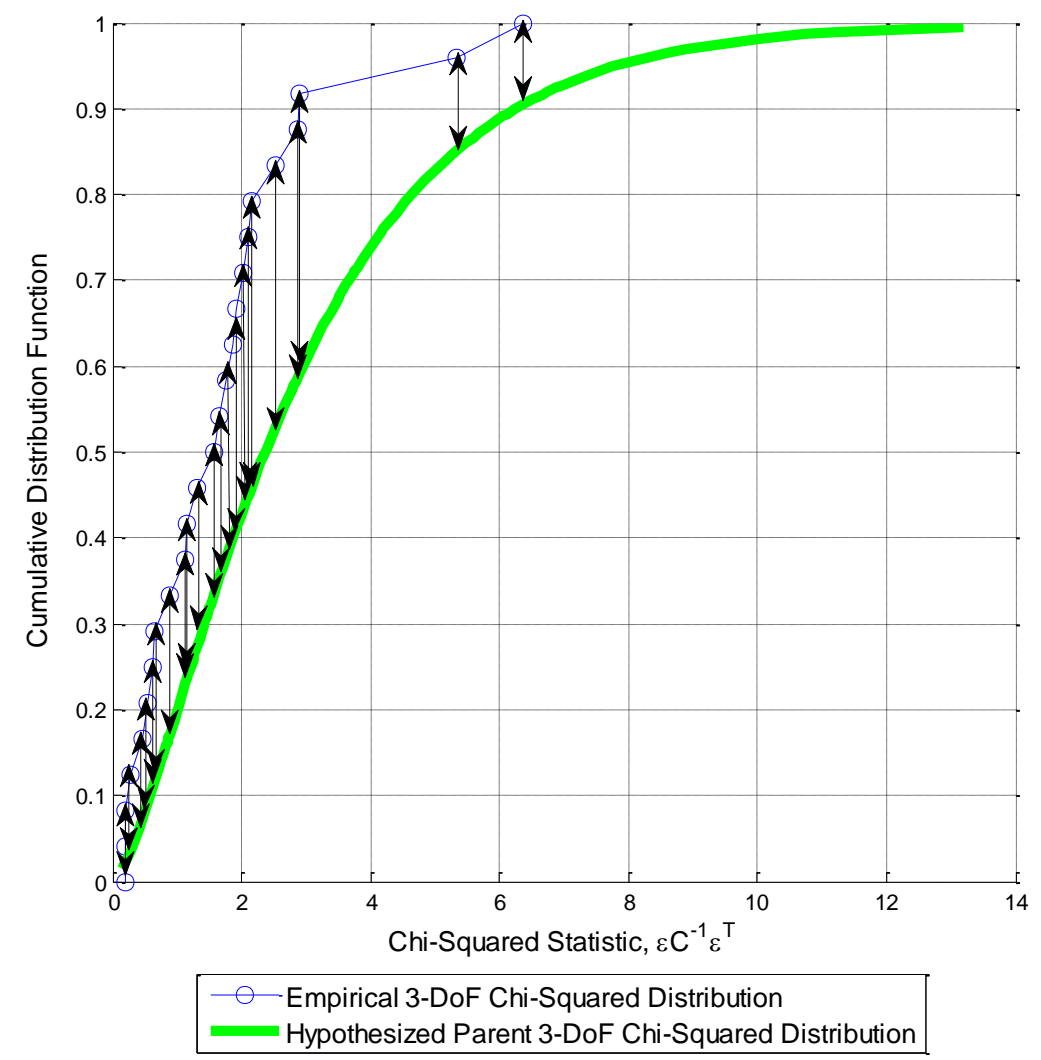

Figure 3. Deviation between empirical and hypothesized parent 3-DoF chi-squared distributions. The blue and green lines represent the empirical and hypothesized parent distributions, respectively. The black double-arrows represent the deviations between the two distributions.

Since a covariance represents an uncertainty in the knowledge of the estimated trajectory, its adequacy as a representation can be evaluated only by examining a group of error data and determining whether these data conform to the distribution specified by that covariance. To this end, the evaluation procedure collects bins of 3-DoF chi-squared statistics at each 60-second propagation point for a group of propagations. These groups of trajectory propagations are each created with controlled amount of RIC acceleration variances per Eq. (4). Accordingly, the number of 3-DoF chi-squared statistics in each bin is equal to the number of trajectories propagated in the group being tested. Ultimately, each bin of 3-DoF chi-squared statistics (at a common propagation point) is tested for realism using the aforementioned ECDF test statistic. Figure 4 shows a $p$-value versus propagation time bar chart in which each $p$-value represents the likelihood the bin of covariances at a particular propagation point conforms to a 3-DoF chi-squared distribution. The covariance realism assessment in this study collects bins at each 60 second propagation time step over a 3.5 day propagation timespan. As a result, 5,040 bins are assessed for covariance realism. However, all $p$-value bar charts in this paper display a thinned amount of bins even though the amount of covariance realism is assessed using the entire set of bins. Because $p$-value tables for different values of $Q$ from Eq. (6) are tabulated from only $1 \%$ through $25 \%$ in $1 \%$ increments, a $26 \% p$-value indicates an off-the-charts superior result, whereas a $0.9 \% p$-value requires a conclusion of non-conformity between the empirical and hypothesized parent distributions. The analyst can use a plot similar to the one presented in Figure 4 to determine in which parts of the propagation timespan the covariance realism is faring well and in which it is faring poorly. 


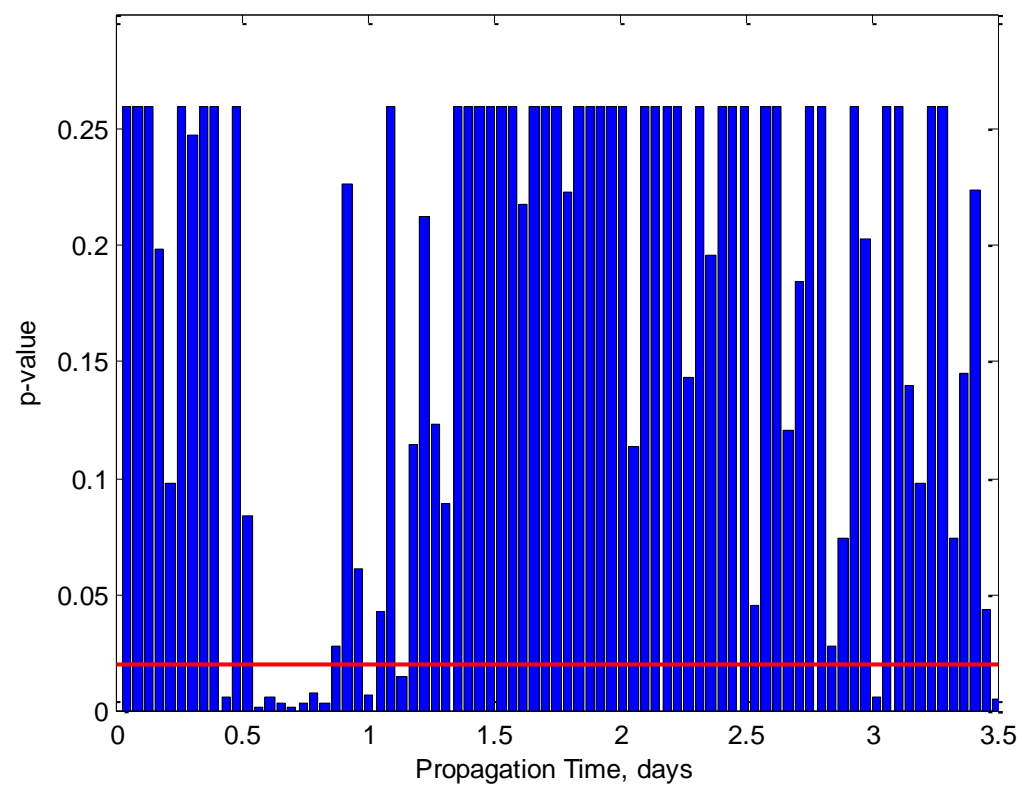

Figure 4. $p$-value vs. Propagation Time chart. A $26 \%$-value represents an "off-the-chart" superior result. The red line represents the $2 \% p$-value threshold or confidence interval. This particular set of trajectories fares well in covariance realism overall but struggles in the 0.5 to 1-day interval.

If covariance realism deficiencies can truly be modeled by static acceleration variances in the process noise context, tuning activities should produce a realistic covariance throughout the entire prediction interval. As a result, frequently when performing covariance realism remediation, an analyst must decide over which portion of the prediction interval they should tune the covariance. The Aqua and Aura covariances are tuned to a 3.5-day propagation timespan (the first 0.5 days of which are not used in the conjunction assessment screening process) because ESMO begins to work a conjunction actively by holding Risk Mitigation Maneuver (RMM) meetings and securing ground station contacts starting 3 days prior to the TCA.

A predicted state estimate is an estimate of a nominal state (i.e., mean position and velocity), and a covariance is the stochastic characterization of the uncertainty in the knowledge of this state. The covariance realism assessment process is based on the assumption that individual component propagation errors are normally distributed. This condition allows a covariance matrix to represent the trajectory propagation error distribution without requiring higher-order moments. As a result, the covariance realism assessment and compensation method in this study determines how well predicted trajectory propagation errors conform to the Gaussian distribution specified by the covariance.

As a result, the following can then be said for each moment of distribution with respect to the covariance: (1) The first moment of the distribution of normalized errors, or the mean, gives the analyst an initial idea of whether the situation is biased. Individual component propagation errors that conform to a Gaussian distribution should have a mean equal to $0,(2)$ The second moment of the distribution, or the standard deviation, of the individual component propagation error distributions. Since the component errors were divided by the expected standard deviations of their distributions, if the covariance standard deviations are representative the result should be a normalized Gaussian random variables (or "z-variables") with a normalized standard deviation thus equal to unity. Values less than one indicate an oversized covariance and greater than one an undersized covariance; the latter situation is far more common in practice, and (3) For a Gaussian distribution, the third and fourth moments (skewness and kurtosis) are defined to be 0 and 3, respectively. Failure to achieve these values in a given distribution can indicate one of two things: the presence of outlier data in the sample or, more seriously, the failure of the data to conform to the expected Gaussian distribution. It is the expectation that, through judicious outlier identification and exclusion, Gaussian behavior can be achieved.

\section{Outlier Trajectory Propagation Identification}

As stated previously, outlier data are frequently present in predicted covariances because of the unpredictability of persistently high or extreme solar activity. Thus, it is important to be able to identify such situations and treat 
outliers accordingly, as they pollute the covariance realism assessment and compensation process. A common choice is the Grubbs outlier test, which provides a formal statistical test for outliers but works only in situations with a single outlier. ${ }^{8}$ A better alternative for the present situation is the Rosner Outlier Test, which can accommodate multiplier outliers in the same sample but requires an a priori guess at the actual outlier candidates. ${ }^{5,6}$ In fact, the test detects outliers that exceed by a stated likelihood what would be expected for the distribution given the particular sample size, and is designed to avoid the problem of masking, where a pair of outliers can each "mask" the other's presence and thus pass undetected by an outlier test. Regarding the a priori guess of potential outliers, the analyst should pay particular attention to the in-track trajectory propagation errors at the final propagation point and testing those trajectories with the largest Root-Mean-Square (RMS) errors. Figure 5 depicts trajectories that appear to be outliers and therefore could be tested. At this time, the input to the Rosner Outlier Test is limited to four potential outliers. Therefore, if more than four potential outliers are determined, only the four most deviant potential outliers are passed through the outlier identification test.

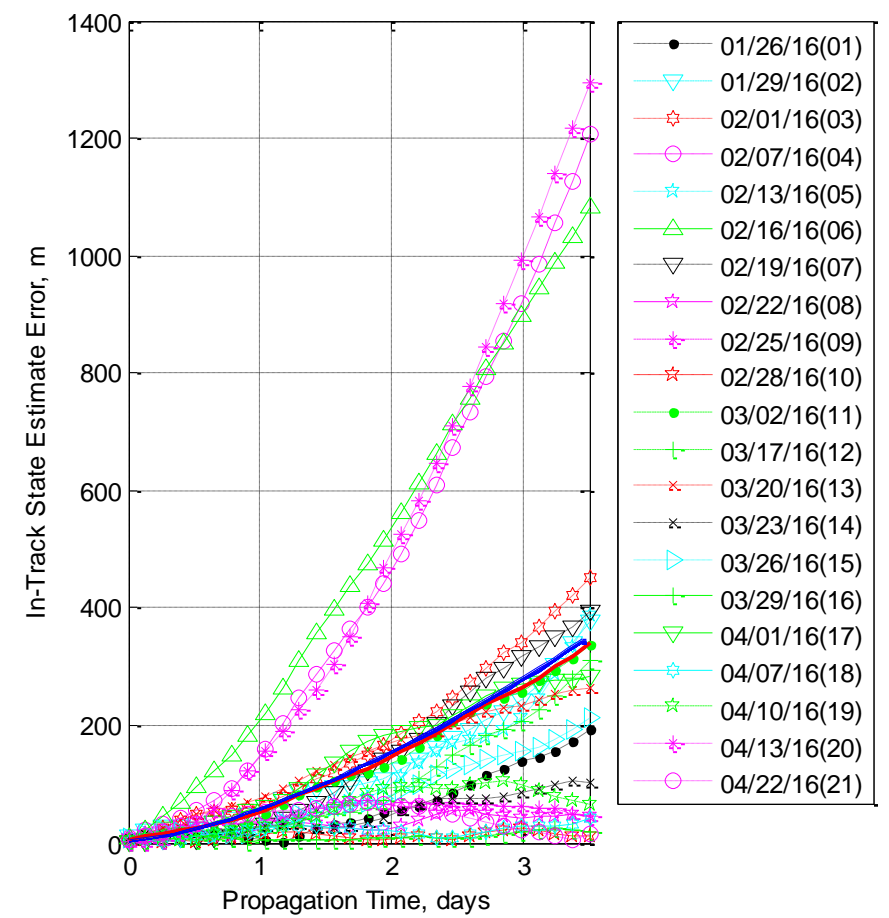

Figure 5. Potential outlier propagations amongst in-track standard errors. The blue curves represent the covariances of each trajectory whereas the red curve represents the mean RMS trajectory propagation error. The 02/07/16 (04), 02/16/16 (06) and 04/13/16 (20) trajectories stand out visually as potential outlier propagations.

The EOS FDS team has adopted a "x-sigma" filter technique in which all trajectories that produce more than \pm 1 standard deviations (from the mean in-track standard component propagation error at the final propagation point) are selected as a priori candidates for the aforementioned Rosner Outlier Test. This method has been working well as of this writing; the daily tuning looks back at the preceding 90 days of covariances and has been consistently identifying the same outliers. Figure 6 depicts the $1 \sigma$ in-track standard error boundaries of well-tuned covariances; those trajectories that lie outside it should be tested for outlier removal. 


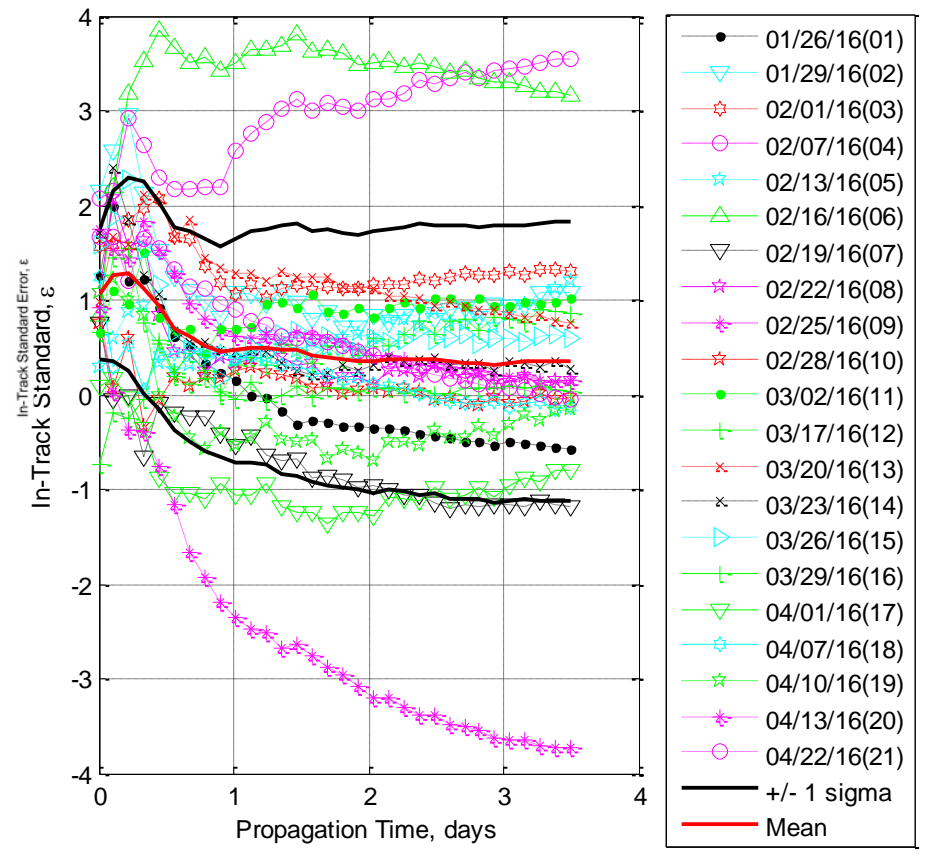

Figure 6. Potential outlier propagations amongst in-track standard errors. The 02/07/16 (04), 02/16/16 (06) and 04/13/16 (20) trajectories are identified as potential outlier propagations again but 02/19/16 (07) is added to the outlier identification test since it lies outside the $1 \sigma$ bound.

\section{Covariance Realism Assessment and Compensation Example}

This section describes the Aqua covariance realism assessment and compensation exercise performed across a August 02, 2014, to November 06, 2014, tuning timespan. The procedure to perform this exercise is provided in the Appendix section for reference. The exercise removes any maneuvers present during this timespan, as those trajectories including maneuvers skew the mean RMS trajectory propagation error that is ultimately used to size the set of covariances. Furthermore, the study uses a 3-day gap between trajectory propagation start dates; this step assures the definitive epoch covariances from which the predicted covariances are being propagated, are independent and free of correlation from one another (since the 2.5 days worth of TDRS and GN observations inserted into daily FDF smoothed OD). With that said, the exercise assumes the predicted covariances were created using the set of controlled values of RIC acceleration variances and $C_{D}$ variance given below:

$$
\bar{q}_{a c c}=\left[\begin{array}{l}
1 \times 10^{-10} \mathrm{~km} / \mathrm{sec}^{2} \\
1 \times 10^{-10} \mathrm{~km} / \mathrm{sec}^{2} \\
1 \times 10^{-10} \mathrm{~km} / \mathrm{sec}^{2}
\end{array}\right]^{2}, Q_{\mathrm{C}_{\mathrm{D}}}=5 \times 10^{-13}
$$

The covariances propagated using the acceleration variances listed in Eq. (7) are severely out-of-tune (less than $5 \%$ of the 3-DoF chi-squared bins are passing the confidence interval over the 3.5 propagation timespan) and in need of careful remediation. In this respect, the analysis presented in this section assumes a definitive orbit is available to the analyst. The predicted component trajectory propagation errors for the August 02, 2014, to November 06, 2014, covariance realism assessment timespan appear in Figure 7. The 10/22/14 and 10/25/14 trajectories readily reveal themselves as potential outlier propagations. This study uses the mean RMS component trajectory propagation errors (after outlier propagation removal) to determine the size of the covariances during the tuning stage. The mean RMS component propagation errors of each group of trajectories are plotted on Figure 7 as well (dark red line). 


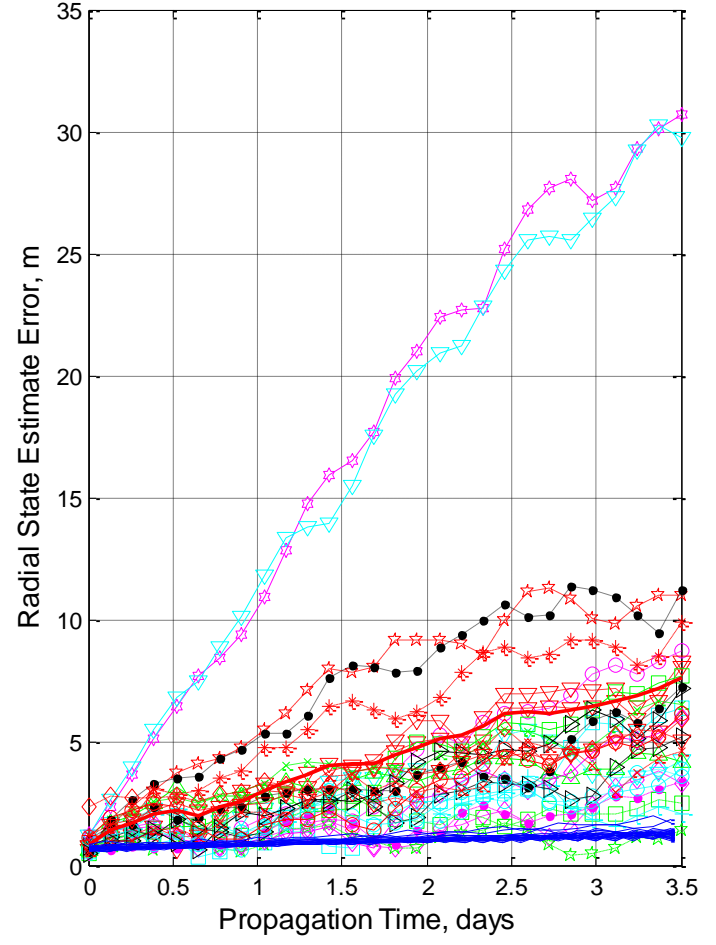

(a)

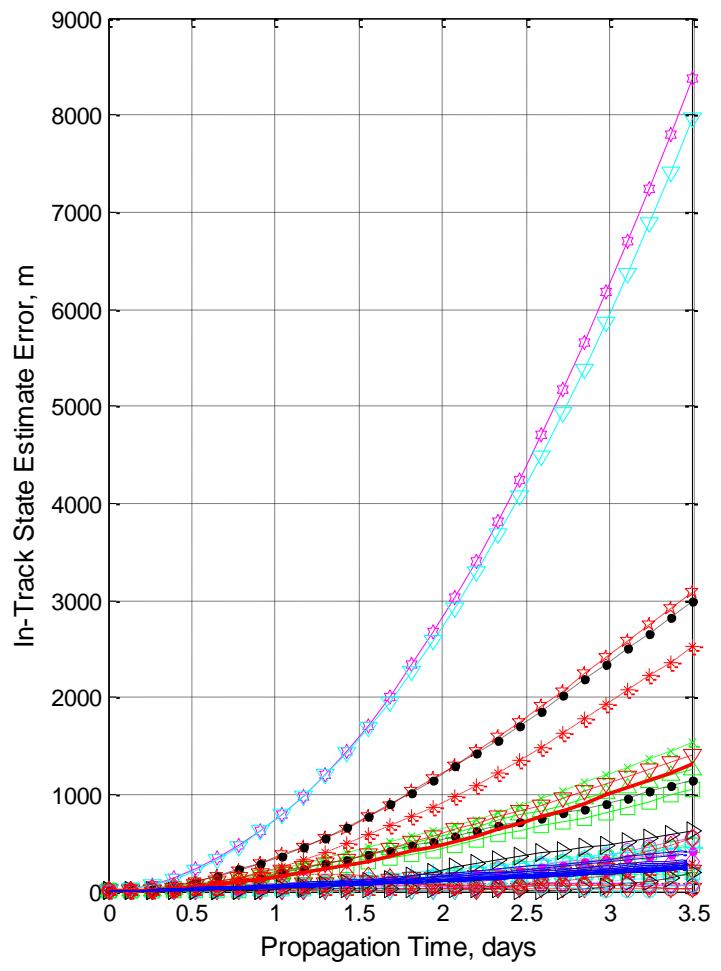

(b)

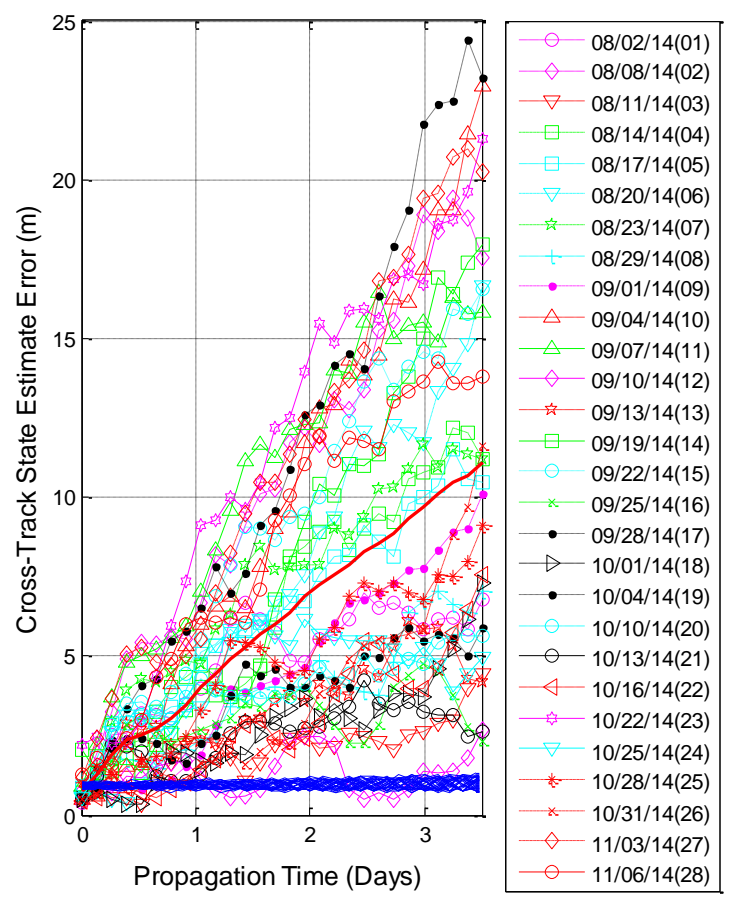

(c)

Figure 7. Component mean RMS and predicted state estimate errors (prior to outlier trajectory removal and covariance tuning). The blue curves represent the trajectories' covariances whereas the red curves represent the mean component RMS errors. The 10/22/14 (23) and 10/25/16 (24) trajectories in plots (a) and (b) are immediately identified as potential outlier trajectory propagations.

12

American Institute of Aeronautics and Astronautics 
The standard component trajectory propagation errors prior to outlier trajectory removal and covariance tuning are plotted in Figure 8. If the correlation terms are small, these errors should reasonably conform to Gaussian distributions and have zero means and standard deviations of 1 and thus give a general indication of the situation, but out-of-tune covariances and the presence of outliers will produce non-Gaussian distributions. More specifically, although the out-of-tune cross-track covariances in Figure 8 have zero mean, their standard deviations are greater than one and thus the covariances are not adequately representing the mean RMS trajectory propagation errors in Figure 7. Furthermore, the presence of potential outliers is adding bias to the means of the radial and in-track covariances in Figure 8.

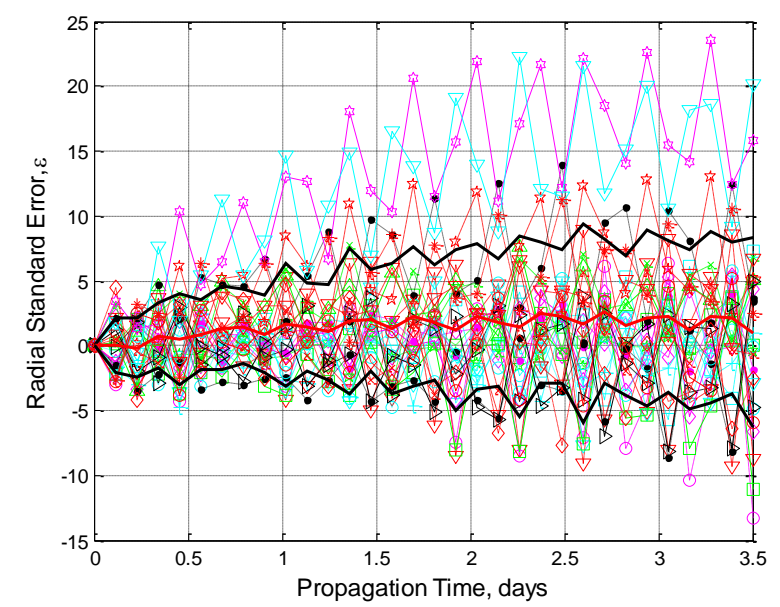

(a)

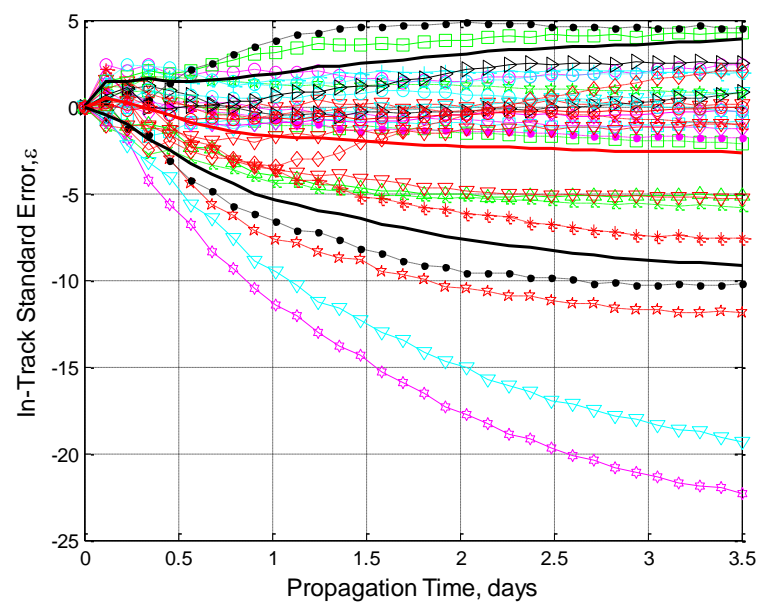

(b)

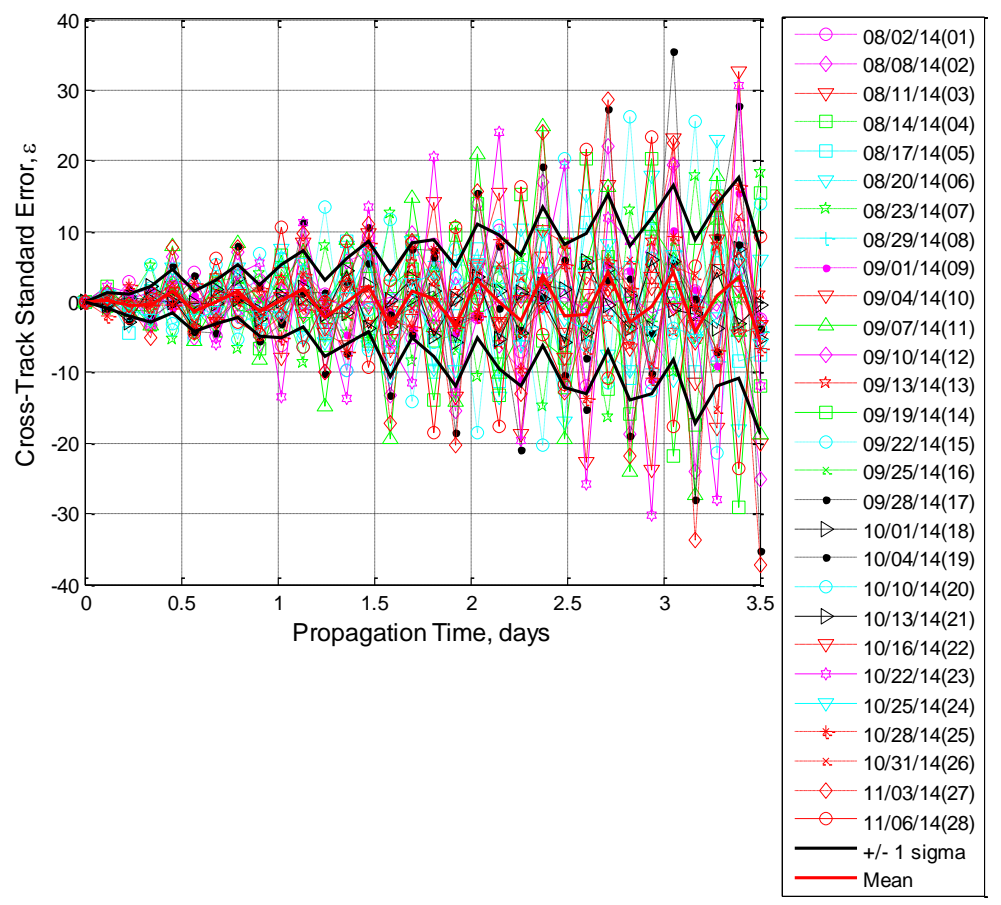

(c)

Figure 8. Standard Component Error vs Propagation Time (prior to outlier trajectory removal and covariance tuning). The errors in plots (a) and (b) do not have a mean equal to 0 nor a standard deviation equal to 1 . The errors in plot (c) have a mean equal to 0 and a standard deviation greater than 1 . Since the $09 / 13 / 14(13), 09 / 28 / 14(17), 08 / 14 / 2014(04)$, and 10/04/14 (19) trajectories are outside the $1 \sigma$ bound at the final propagation point in plot (b), they have been added to the outlier identification test. 
The empirical vs. hypothesized parent normal CDF distributions for the bins of standard component errors prior to outlier trajectory removal and covariance tuning are plotted in Figure 9. Each plot is confirming the covariances are out-of-tune with potential outliers present in the radial and in-track directions.

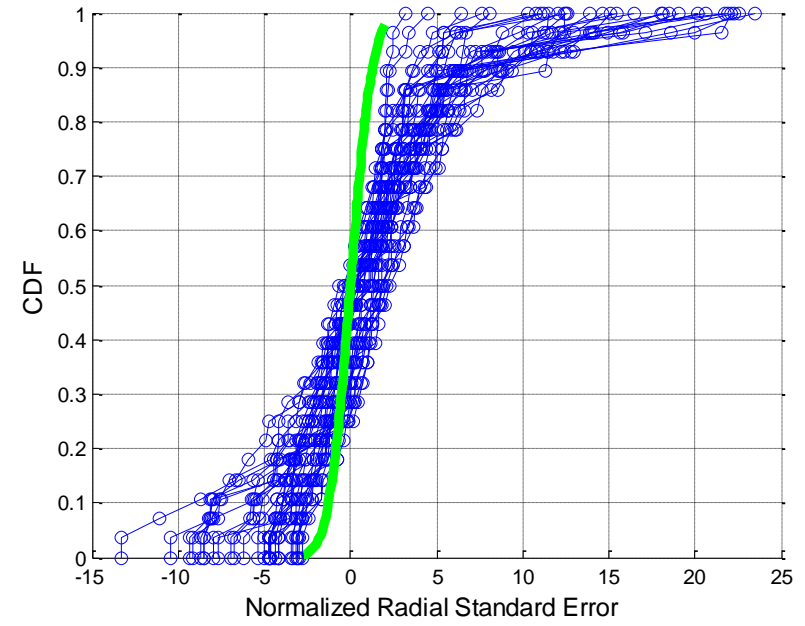

(a)

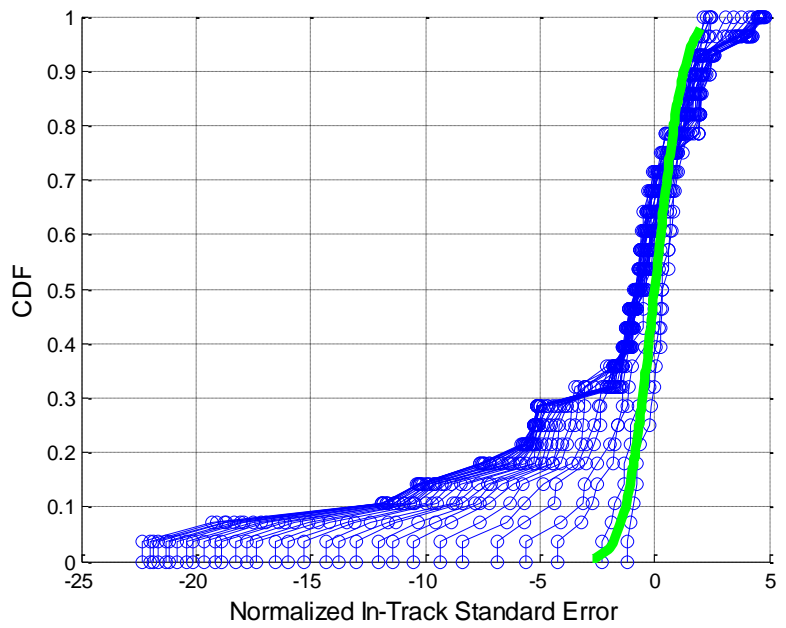

(b)

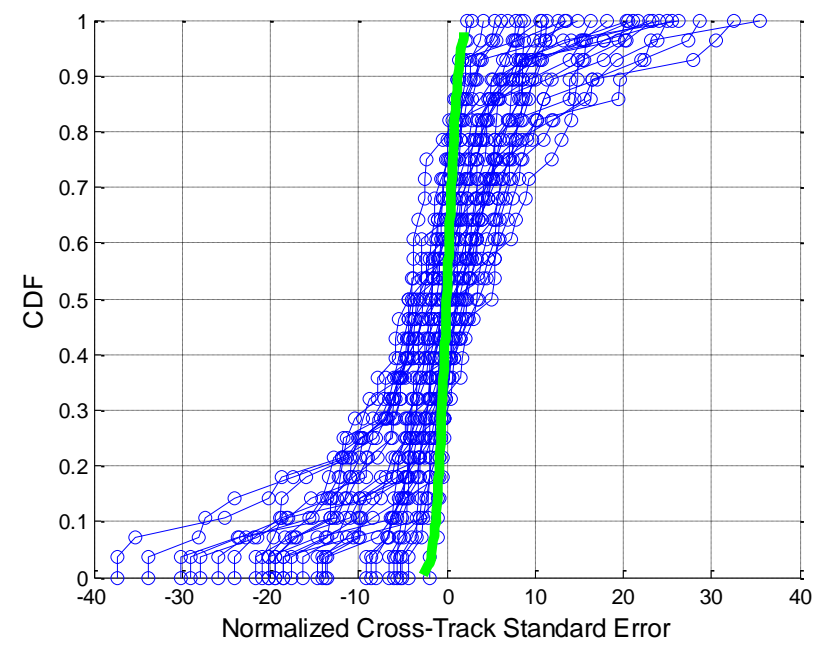

(c)

Figure 9. Standard Component Error vs Standard Component Error CDFs (prior to outlier trajectory removal and covariance tuning). The blue curves represent empirical distributions at each propagation point whereas the green curve represents the hypothesized parent distribution. Plots (a) and (b) indicate the presence of outlier trajectories and out-of-tune covariances.

Additionally, the empirical verses hypothesized parent $3 \mathrm{CDF}$ distributions for the bins of 3-DoF chi-squared statistics appear in Figure 10. The heavy upper tail distribution is indicative of outlier trajectory propagations and an out-of-tune set of covariances. Once outlier trajectory propagations are removed and tuning of the covariance is complete, the empirical distributions in Error! Reference source not found. and Figure 10 should conform much closer to their hypothesized parent distributions. 


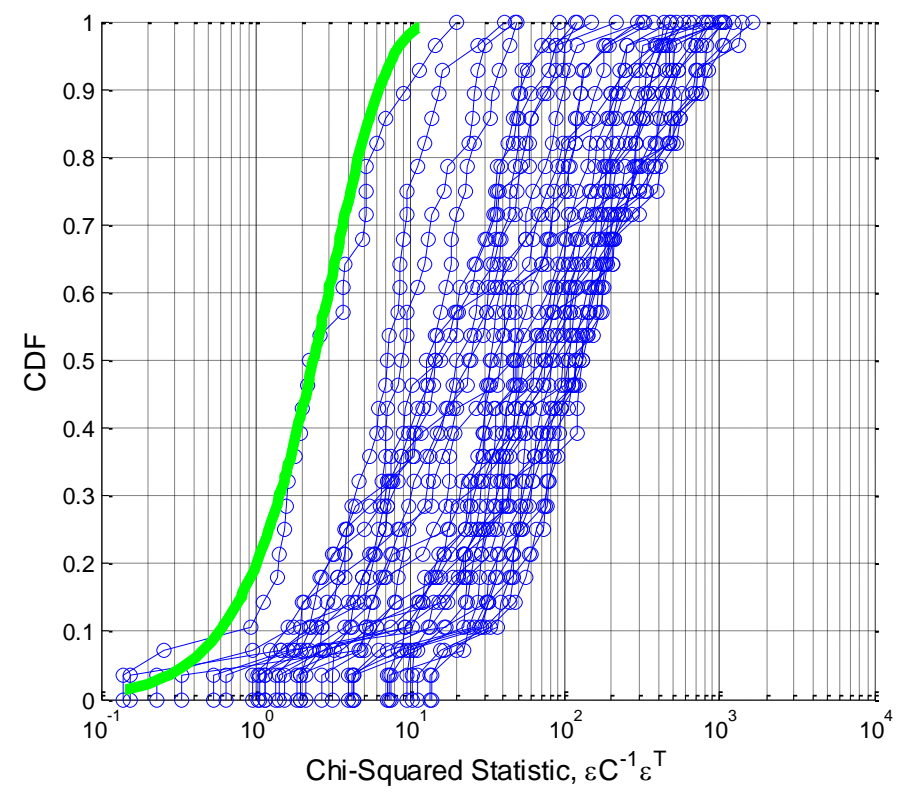

Figure 10. Empirical and hypothesized parent 3-DoF chi-squared distributions (prior to outlier trajectory removal and covariance tuning). The blue curves represent empirical distributions at each propagation point whereas the green curve represents the hypothesized parent distribution.

A sample of $p$-values versus predictive propagation time are plotted in Figure 11. Typically, a Pass Percentage above $80 \%$ is a good result, although the adequacy of the outcome is dependent on where in the propagation interval any non-compliance falls. In this case, we see that, without outlier trajectory removal and tuning, the Pass Percentage is only $2.5 \%$

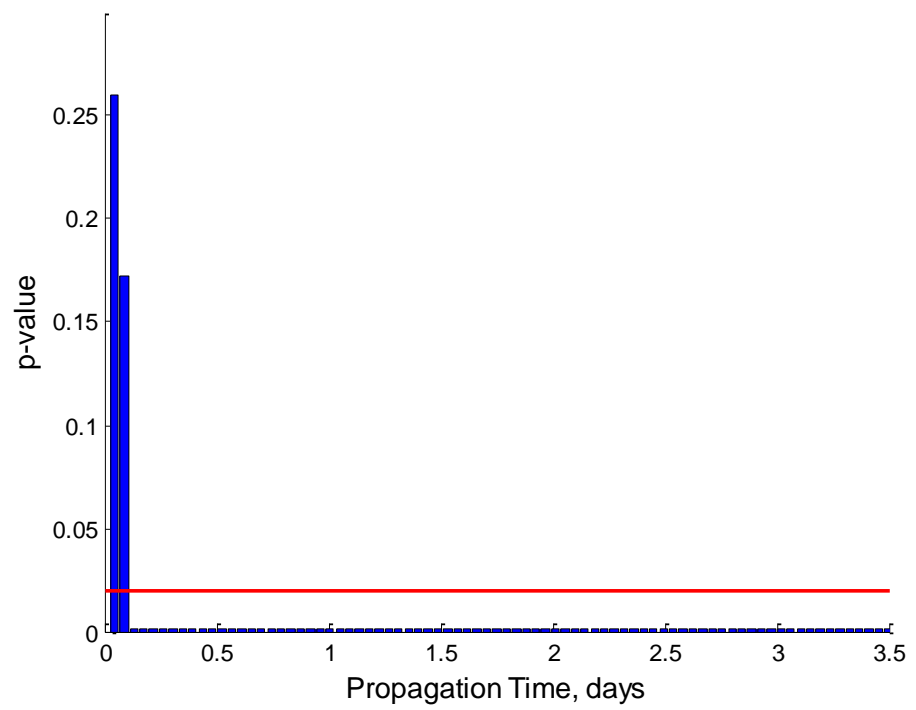

Figure 11. $p$-value vs. Propagation Time bar chart. The red line represents the $2 \%$ Confidence Interval whereas the blue lines each represent the likelihood the empirical distribution conforms to its hypothesized parent distribution at a particular propagation point. Only $2.5 \%$ of the trajectory propagation timespan of the set of trajectories that formed this plot is conforming to a 3-DoF chi-squared hypothesized parent distribution. 
The final propagation point in-track propagation error set (standardized) is the final column of points in Figure 8. The goal is to perform outlier trajectory testing on the normalized values of this group of errors since they are the most pronounced throughout the entire propagation timespan and therefore most likely to reveal themselves as products of outlier trajectories. Six potential outlier trajectories outside the $\pm 1 \sigma$ in-track propagation error bounds are revealed in Figure 12 (although only four can be tested). From most to least deviant, the trajectory propagation dates are 10/22/14, 10/25/14, 09/13/14, 09/28/14, 08/14/2014, and 10/04/14. Table 3 contains the outlier identification results for the August 02, 2014, to November 06, 2014, covariance assessment timespan.

Table 3. Outlier identification test results for the August 02, 2014, to November 06, 2014, Aqua covariance assessment exercise. The 09/13/14, 09/28/14, 10/22/14, and 10/25/14 trajectories are identified as outliers.

\begin{tabular}{|l|c|}
\hline Trajectories Tested for Outlier Removal & $\boldsymbol{p}$-value \\
\hline \hline $10 / 22 / 14$ & 0.0346 \\
\hline $10 / 22 / 14,10 / 25 / 14$ & 0.0000 \\
\hline $10 / 22 / 14,10 / 25 / 14,09 / 13 / 14$ & 0.1100 \\
\hline $10 / 22 / 14,10 / 25 / 14,09 / 13 / 14,09 / 28 / 14$ & 0.0000 \\
\hline
\end{tabular}

The outlier test is run until for all 4 cases and then stopped. The results in Table 3 indicate the 10/22/14, 10/25/14, 09/13/14, and 09/28/14 trajectories are all outliers and can be removed from the analysis. Furthermore, the outlier test revealed a problem alluded to earlier known as masking in which the first and second potential outlier trajectories were identified as outliers but were not outliers when the third potential outlier was added to the group. However, the first three potential outliers were identified as outliers when the fourth potential outlier was added to the group. The Rosner Outlier Identification test is specifically designed to overcome masking. Figure 12 now reveals the same component propagation errors as those in Figure 7 but with the outlier trajectories removed. In Figure 13 we see the bias in the radial and in-track directions has been removed yet the standard deviations are greater than 1; the cross-track directions remains unchanged after outlier trajectory removal. Additionally, we see in Figure 14 the skewness of the radial propagation error distribution and the kurtosis of the in-track propagation error distribution both improved after the outlier trajectories were removed However, Figure 15 and Figure 16 indicate that even after outlier trajectory removal, the 3-DoF chi-squared distributions have not improved. This is expected, as the tuning of the covariance has not been performed. Rather, we have removed outliers to produce a mean RMS trajectory propagation error that we will use to tune the covariances. 


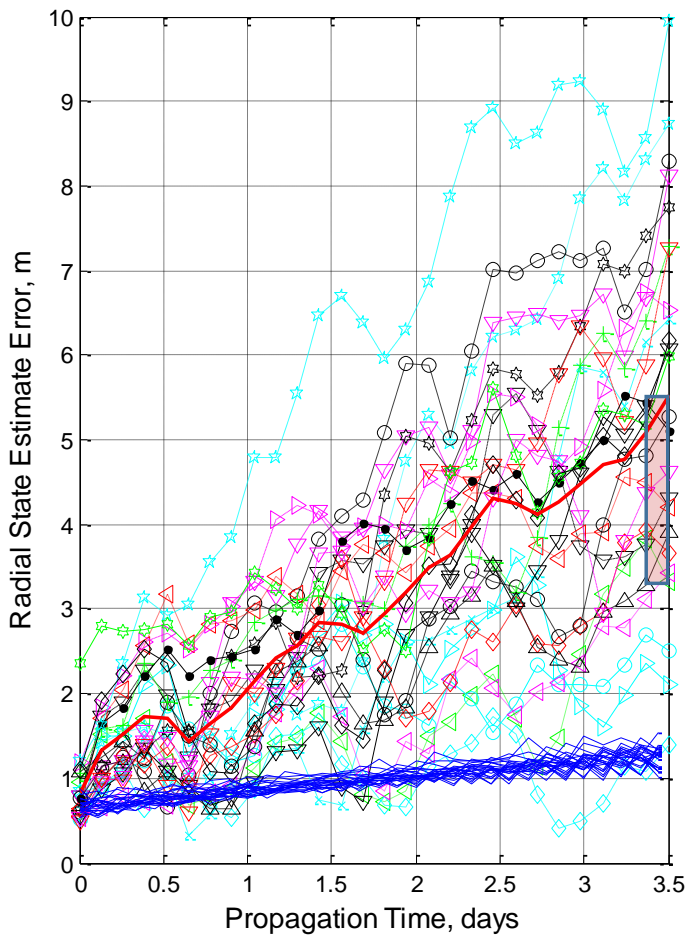

(a)

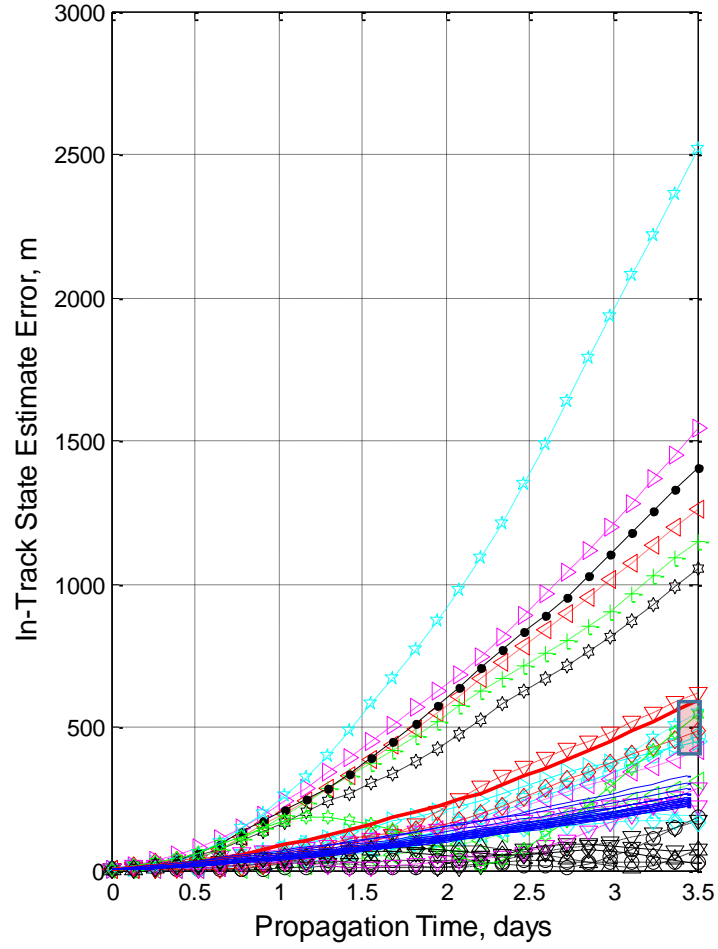

(b)

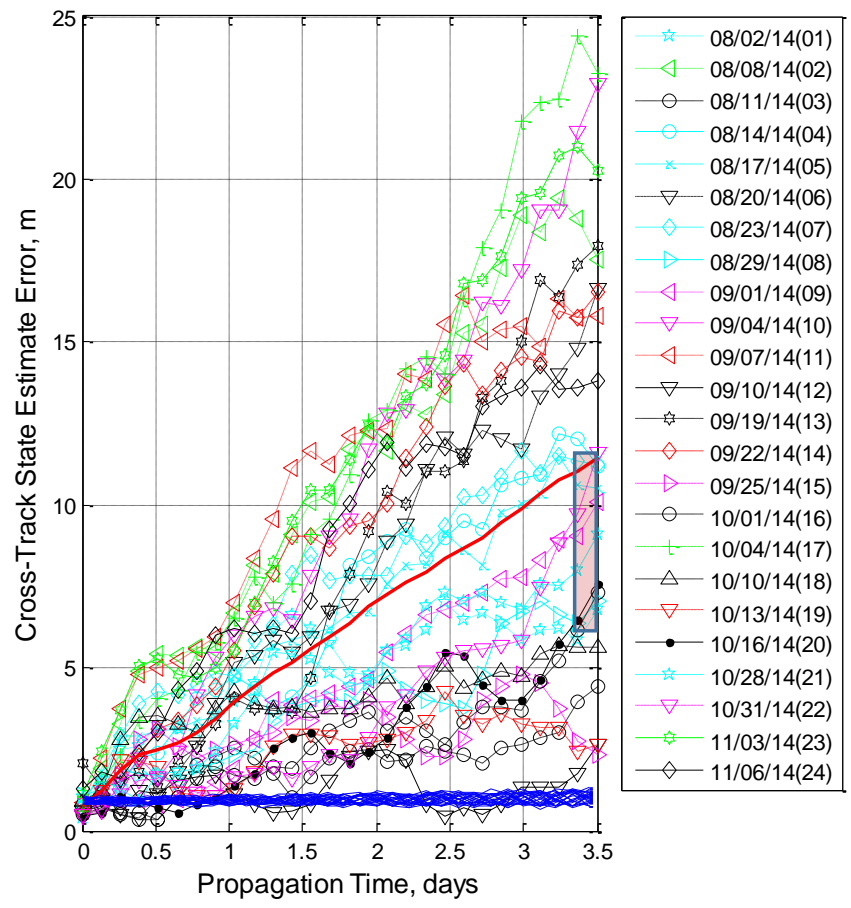

(c)

Figure 12. Component mean RMS and predicted state estimate errors (after outlier trajectory removal and prior to covariance tuning). The blue curves represent the trajectories' covariances whereas the red curves represent the mean component RMS errors. There is potential (depicted by the red transparent bands at the right) to inject process noise into all three components 


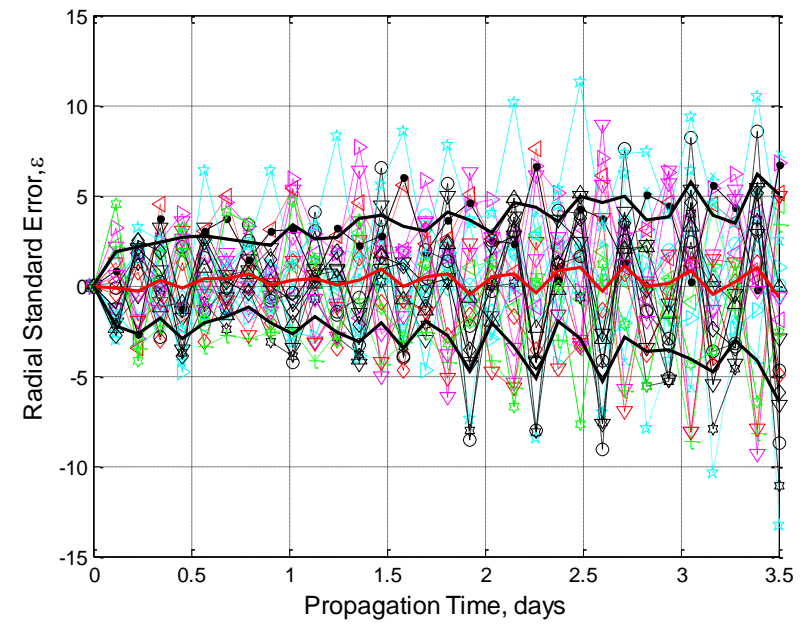

(a)

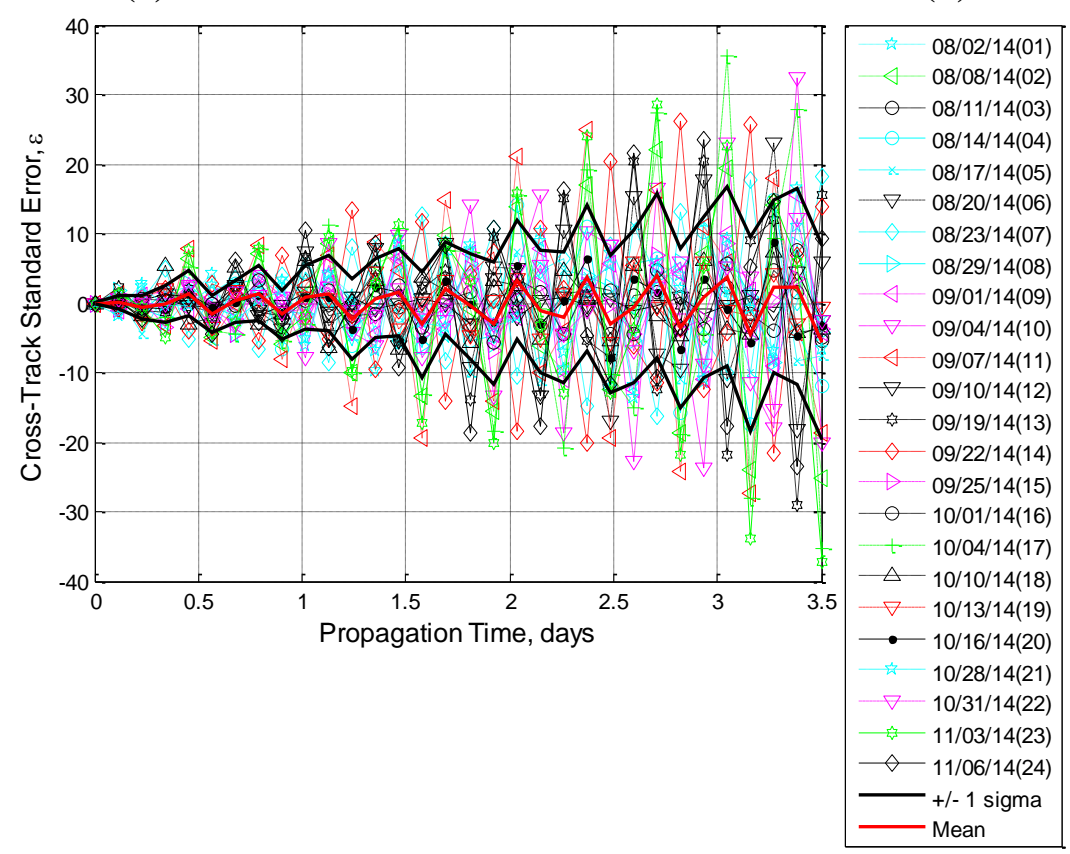

(c)

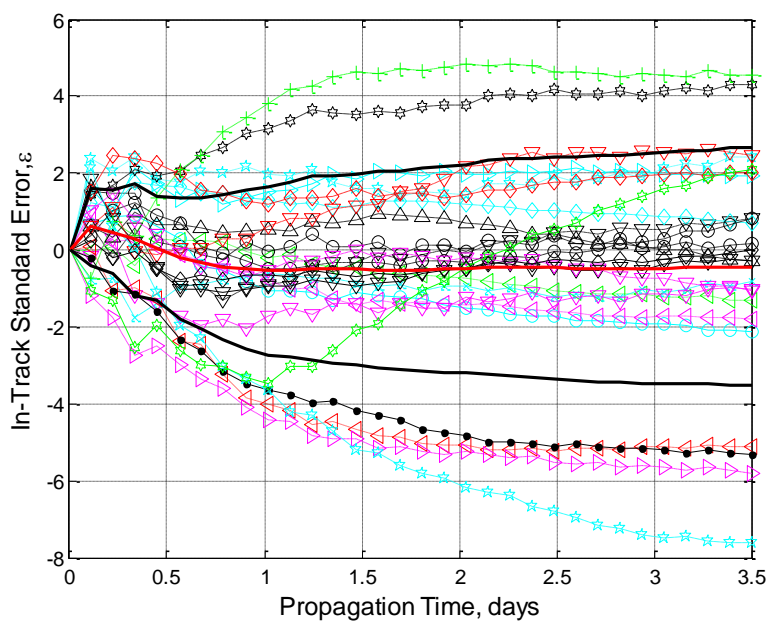

(b)

Figure 13. Standard Component Error vs Propagation Time (after outlier trajectory removal and prior to covariance tuning). The errors in plots (a) and (b) now have means closer to 0 but standard deviations still not equal to 1 . The errors in plot (c) have a mean equal to 0 and a standard deviation greater than 1. 


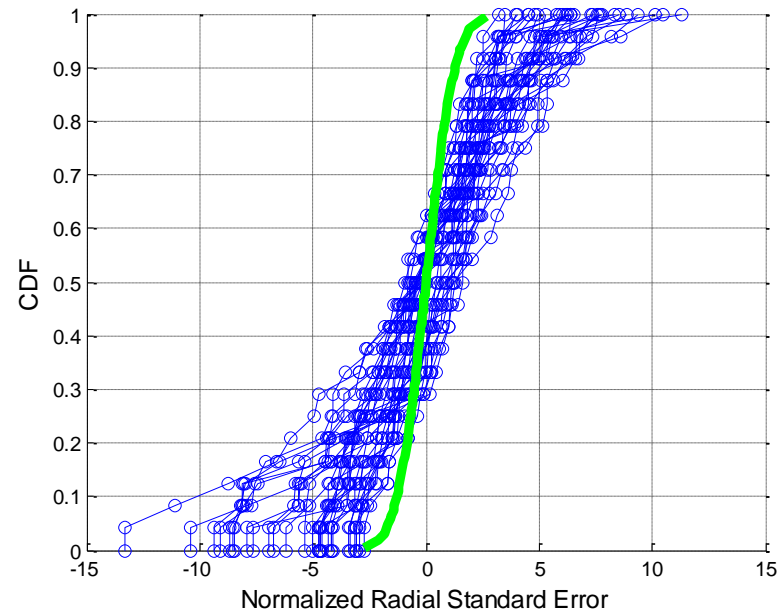

(a)

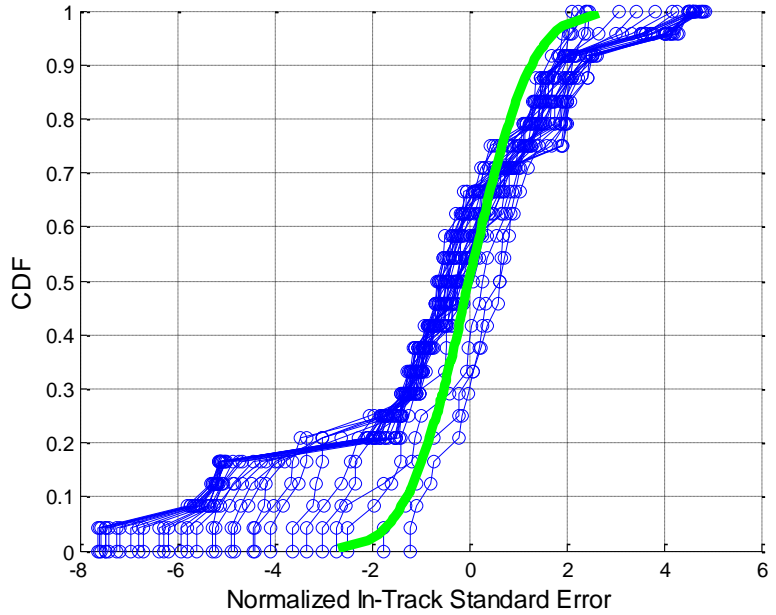

(b)

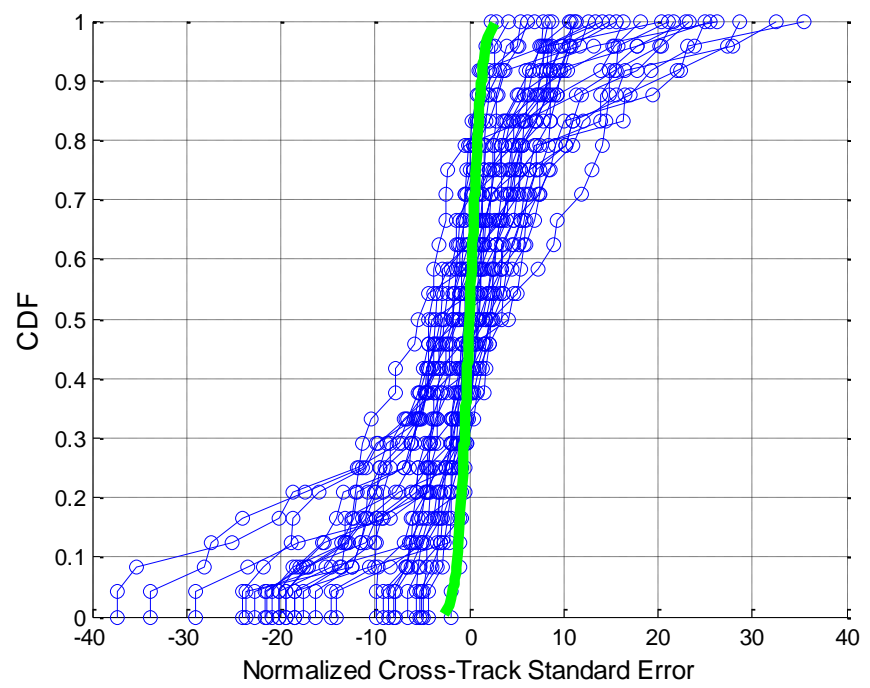

(c)

Figure 14. Standard Component Error vs Standard Component Error CDFs (after outlier trajectory removal and prior to covariance tuning). The blue curves represent empirical distributions at each propagation point whereas the green curve represents the hypothesized parent distribution. Both plots (a) and (b) show improvement in expected behavior in radial and in-track, respectively, after outlier trajectory removal. 


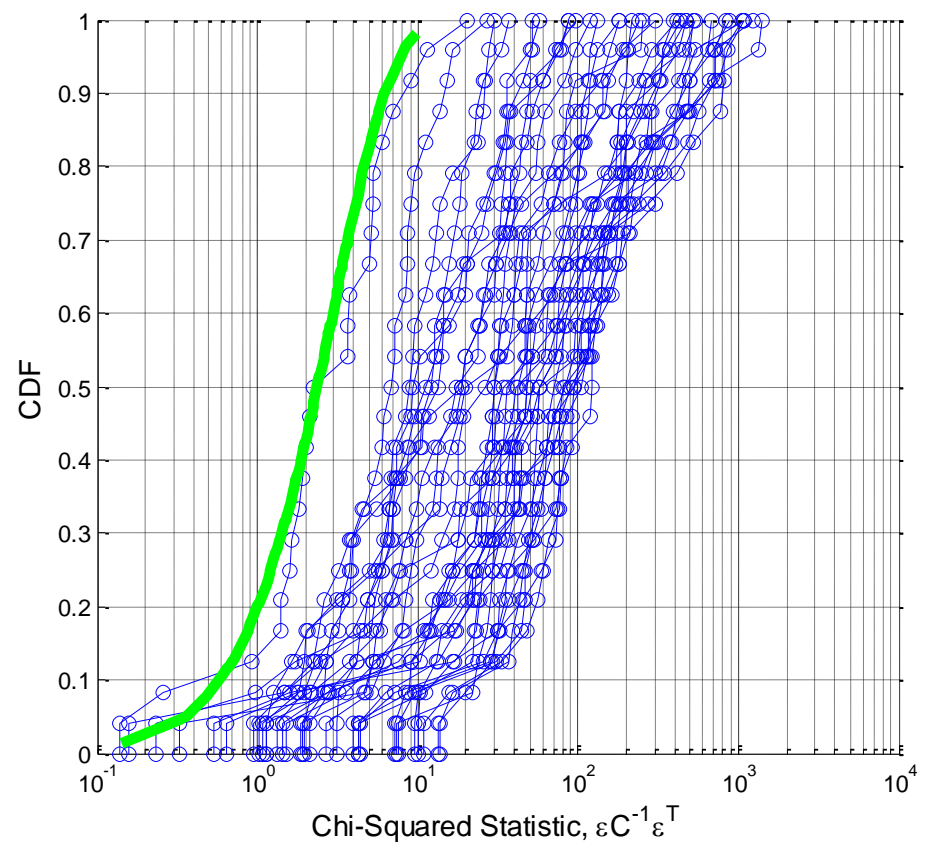

Figure 15. Empirical and hypothesized parent 3-DoF chi-squared distributions (after outlier trajectory removal and prior to covariance tuning). The blue curves represent empirical distributions at each propagation point whereas the green curve represents the hypothesized parent distribution. Outlier trajectory removal has not improved the empirical distributions.

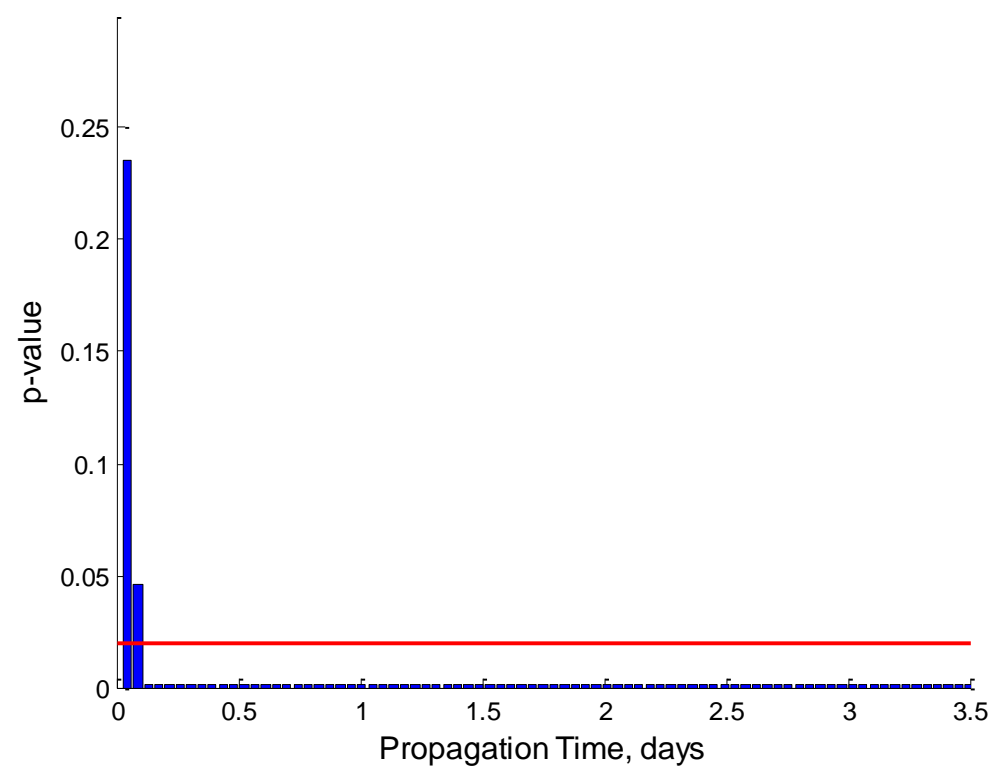

Figure 16. $p$-value vs. Propagation Time bar chart (after outlier trajectory removal and prior to covariance tuning). The red line represents the $2 \%$ Confidence Interval whereas the blue lines each represent the likelihood the empirical distribution conforms to its hypothesized parent distribution at a particular propagation point. Even after outlier trajectory removal, only $2.5 \%$ of the trajectory propagation timespan of the set of trajectories is conforming to a 3-DoF chi-squared hypothesized parent distribution.

Before proceeding to covariance tuning, it is helpful to identify a physical reason for each discarded trajectory's status as an outlier. Naturally, with a propagation error in the in-track and thus radial direction, it is sensible to look 
at the behavior of solar activity on the dates those trajectories were propagated. Based on the solar activity plot in Figure 17, we find the F10.7 flux was persistently high on 3 of the 4 outlier trajectory propagation dates. Furthermore, we find the geomagnetic index was elevated for the fourth outlier trajectory propagation date. With plausible supporting reasoning behind the outlier trajectory removal, the next step is to tune the covariance to the mean RMS propagation error of the updated group of trajectories.

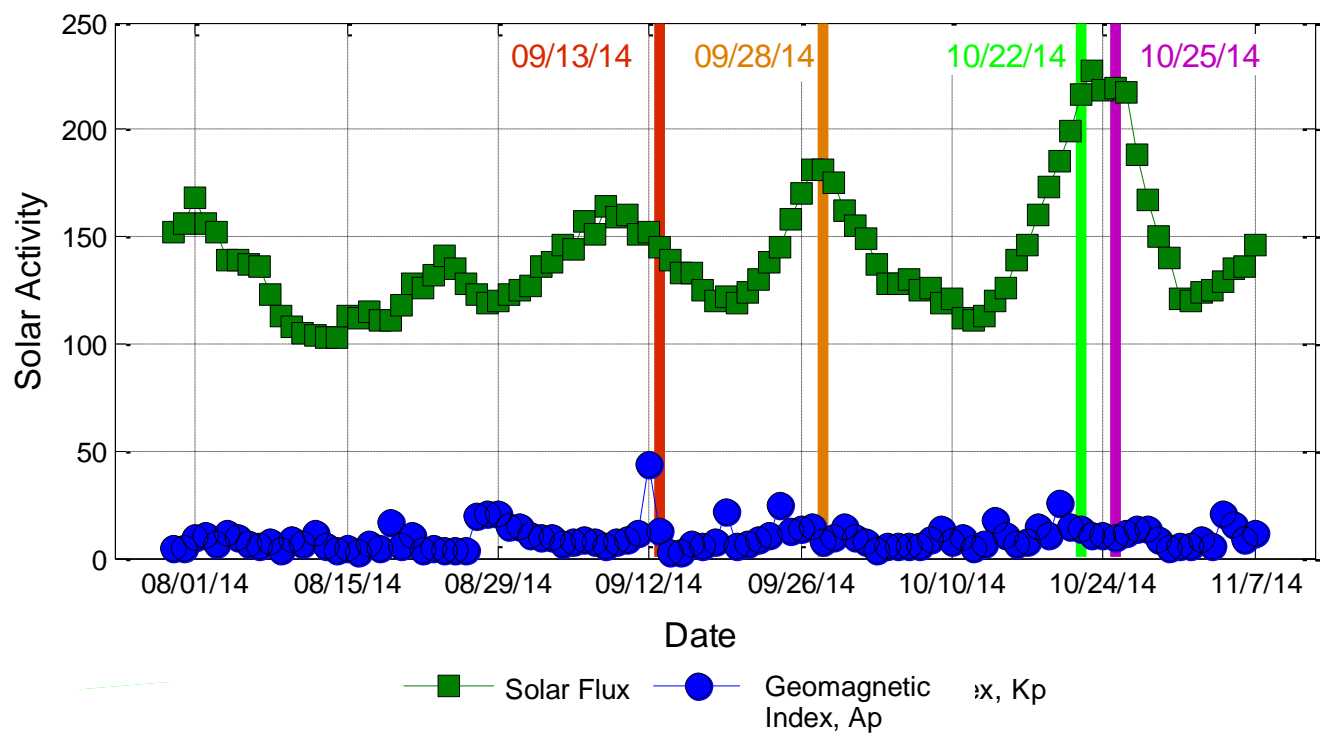

Figure 17. Solar activity between 30 Jul 2014 and 07 Nov 2014 that impacted the density model used by FreeFlyer. The F10.7 Flux exhibited persistent peaks on 09/27/14 and 10/24/14. As a result, the 09/28/14, 10/22/14, and 10/25/14 trajectories failed the outlier identification test around all of these dates. The geomagnetic index exhibited a peak on $09 / 12 / 14$, causing the 09/13/14 trajectory to fail the outlier identification test.

The overall results of this study show that less deviation between the blue predicted covariance and the red mean component RMS propagation error curves in Figure 18 produce high levels of covariance realism. However, this study also shows that it is not possible to make the blue and red curves match at every propagation point due to the following subtleties: (1) force modeling differences in the propagations between the ODTK definitive and FreeFlyer predictive trajectories and (2) the difference in the progression of both curves. Therefore, this procedure attempts to lessen the deviation between the blue covariance and red mean RMS trajectory propagation error curves by decreasing the deviation between their values at the final propagation point in the radial and in-track axes.

On the other hand, due to the logarithmic behavior of the blue cross-track covariance and the nearly linear shape of the red mean RMS cross-track propagation error curve (both appearing in Figure 18), matching the blue and red curves at the final propagation point has an adverse impact on the amount of covariance realism through the entire propagation timespan. Therefore, the blue cross-track curve should not be sized to match the red cross-track curve at the final propagation point. Rather, the amount of cross-track acceleration variance must be added or subtracted until the red and blue curves intersect at the midpoint of the propagation. This technique has been shown to minimize the impact on covariance realism caused by the differences in the shapes of both curves.

As a result, after applying the following acceleration variances,

$$
\bar{q}_{a c c}=\left[\begin{array}{c}
1.7 \times 10^{-9} \mathrm{~km} / \mathrm{sec}^{2} \\
2.5 \times 10^{-10} \mathrm{~km} / \mathrm{sec}^{2} \\
3 \times 10^{-9} \mathrm{~km} / \mathrm{sec}^{2}
\end{array}\right]^{2}, Q_{\mathrm{C}_{\mathrm{D}}}=5 \times 10^{-13}
$$

the Pass Percentage increases from 2.5\% to $86.25 \%$ (as shown in Figure 22). The final scale factors of the rootvariances of the covariance matrix at the 3.5-day propagation point between the covariances prior to and after tuning are 3.5 in the radial direction, 2 in the in-track direction, and 8 in the cross-track direction. Whereas the cross-track 
scale factor is not expected to change (due to well-modeled luni-solar perturbations), the radial and in-track scale factors are sensitive to solar activity and are expected to change through covariance tuning cycles.

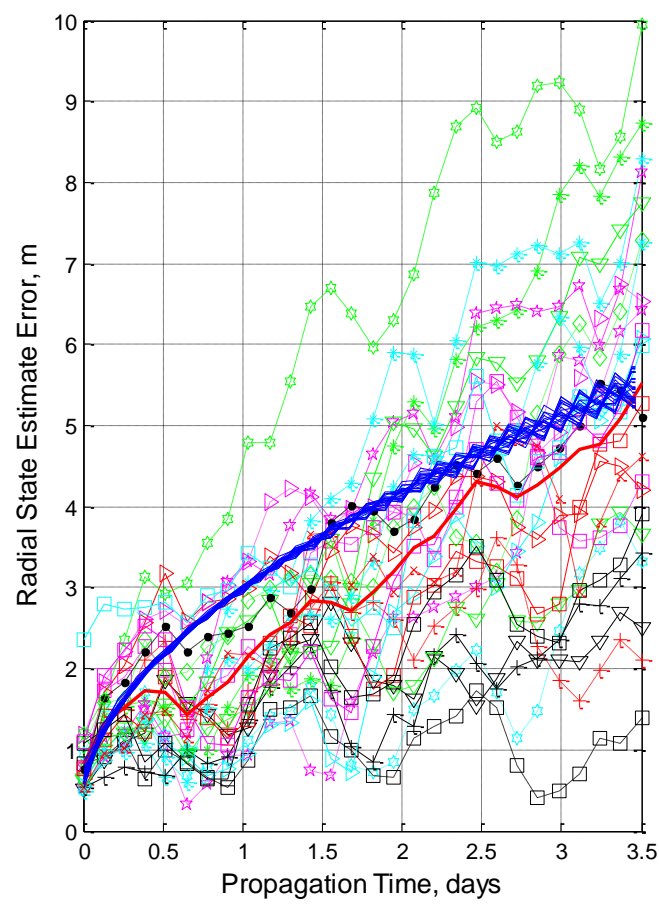

(a)

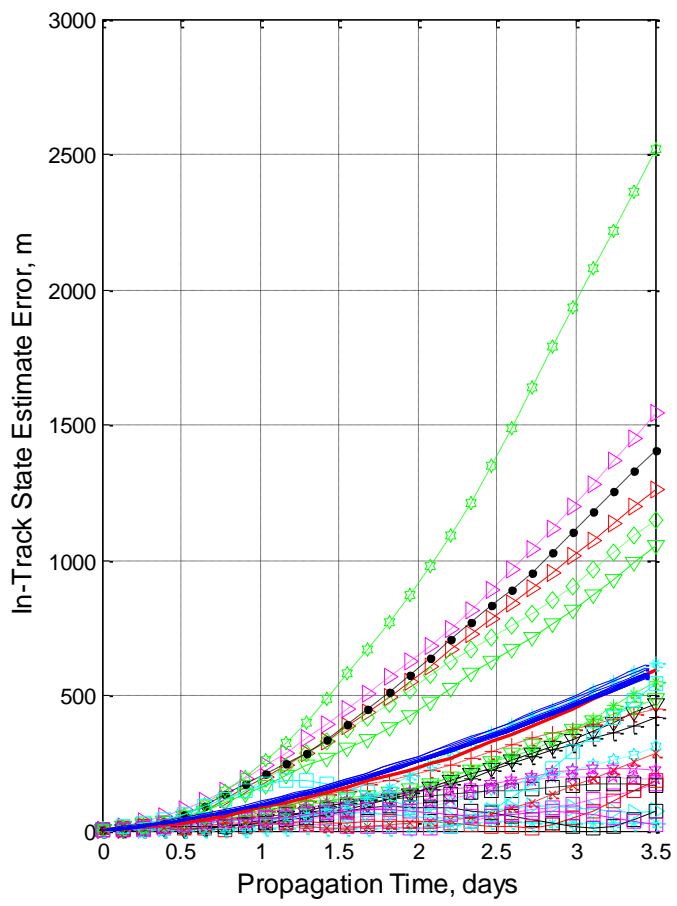

(b)

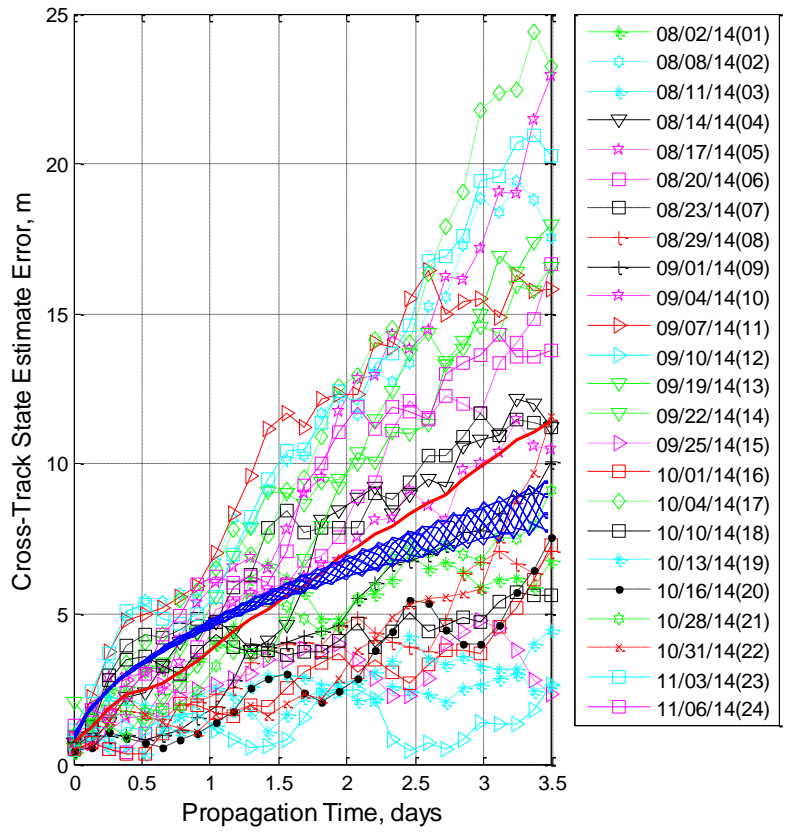

(c)

Figure 18. Component Mean RMS and Predicted State Estimate Errors (after outlier trajectory removal and covariance tuning). The blue curves represent the trajectories'covariances whereas the red curves represent the mean component RMS errors. The radial and in-track covariances are sized to their mean RMS error using the final propagation point and the cross-track covariances are sized to their mean RMS error using the mid propagation point. 


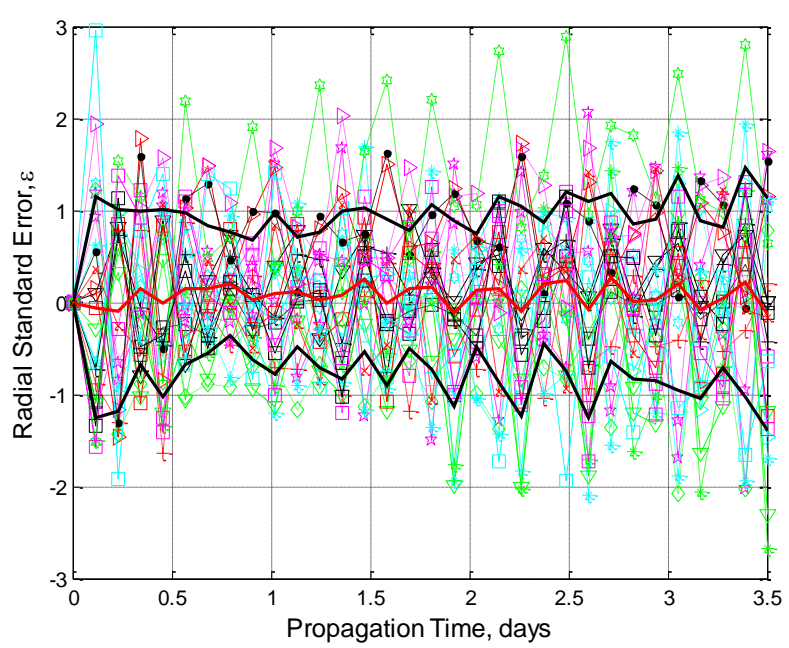

(a)

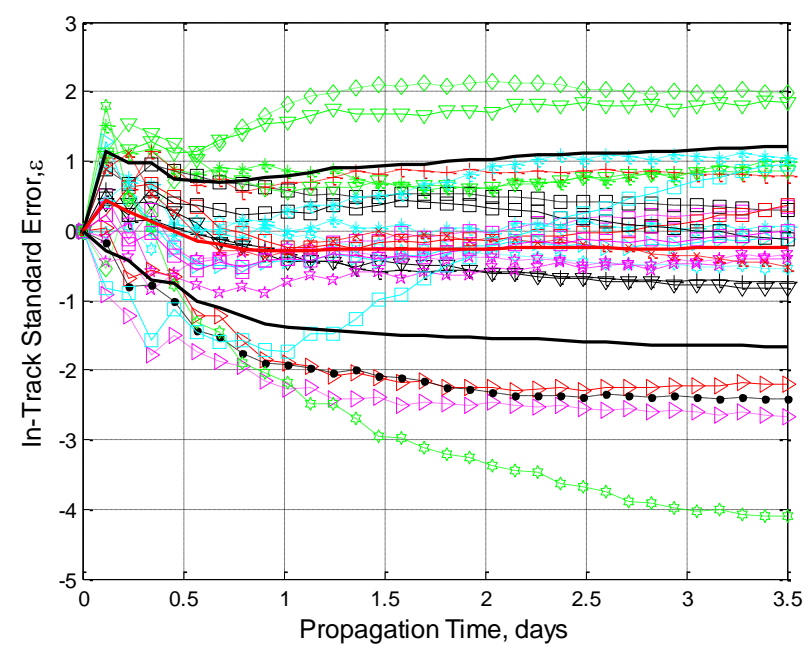

(b)

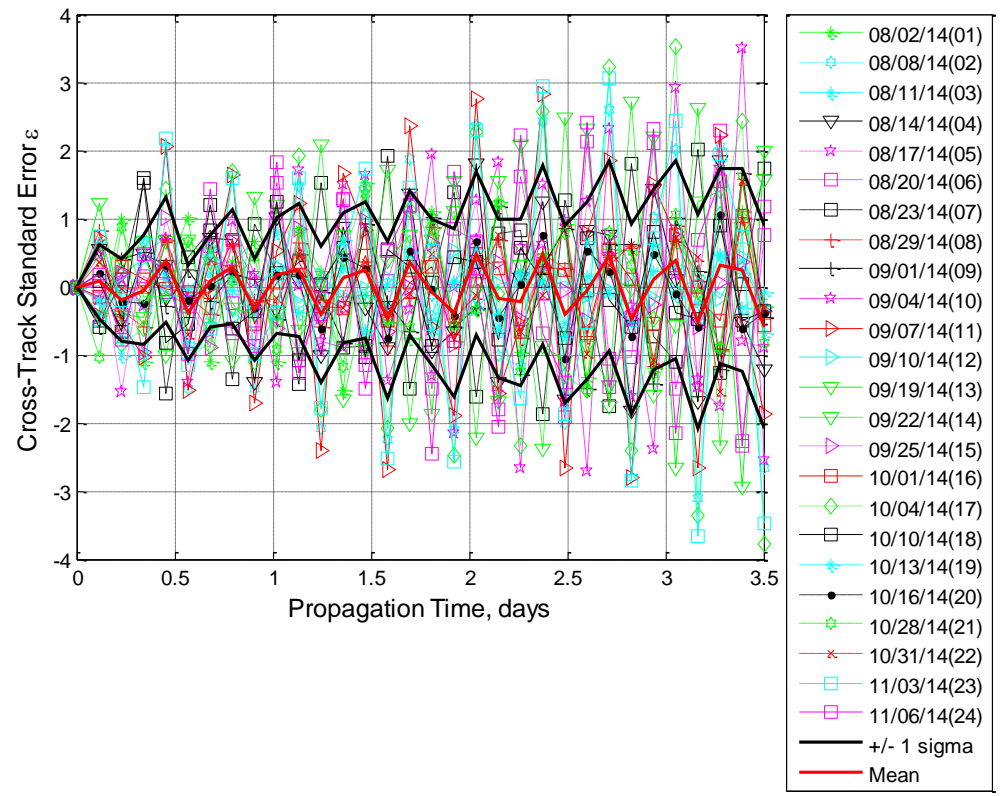

(c)

Figure 19. Standard Component Error vs Propagation Time (after outlier trajectory removal and covariance tuning). The errors in plots (a) and (c) both have means equal to 0 and standard deviations closer to 1 . The errors in plot (b) have a mean closer to 0 and standard deviations greater than but closer to 1 after covariance tuning. 


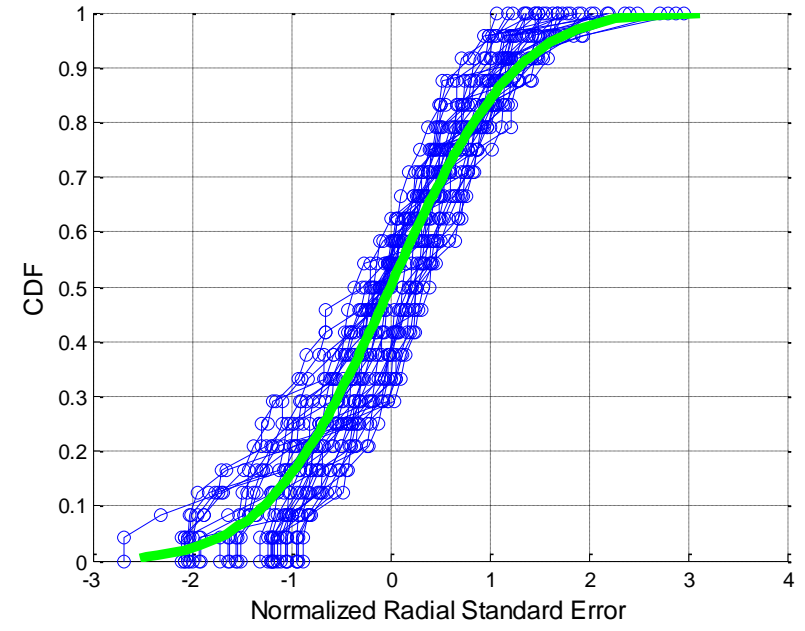

(a)

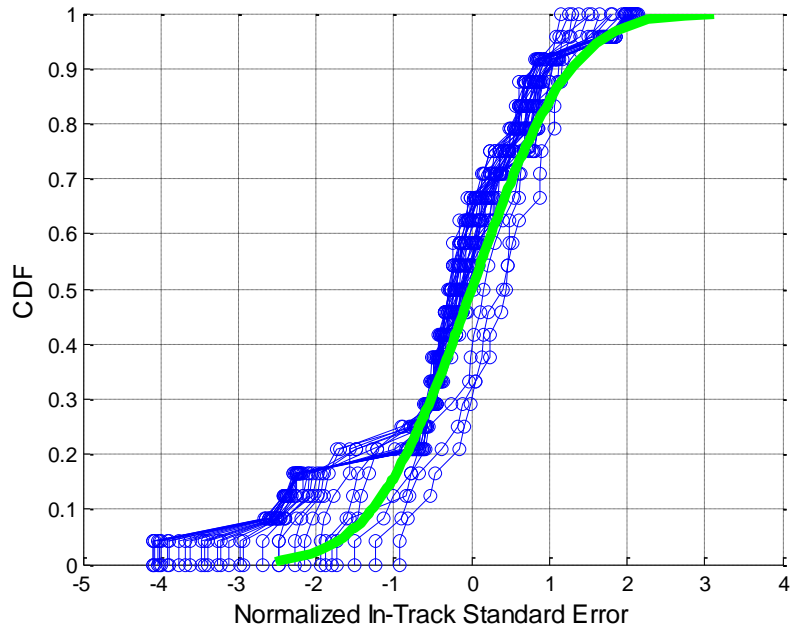

(b)

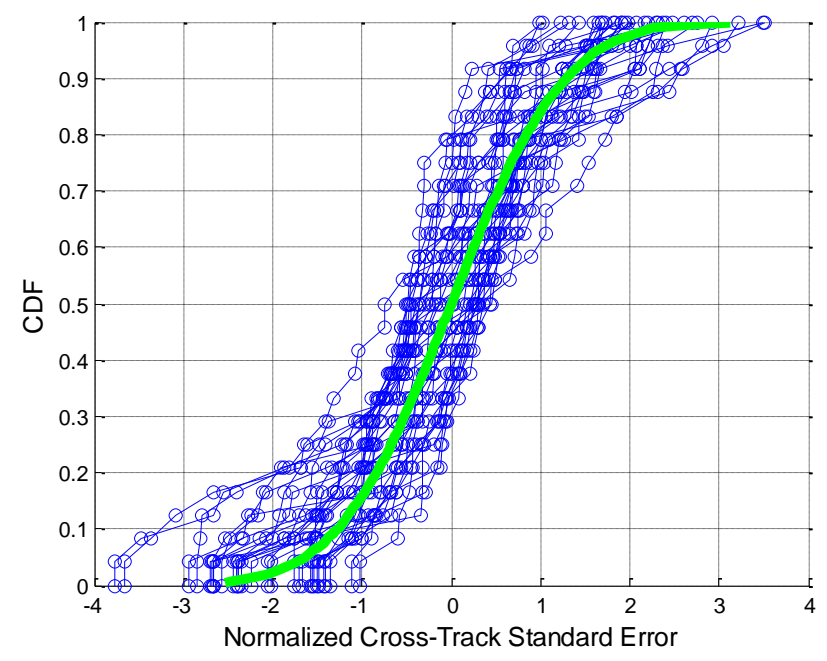

(c)

Figure 20. Standard Component Error vs Standard Component Error CDFs (after outlier trajectory removal and covariance tuning). The blue curves represent empirical distributions at each propagation point whereas the green curve represents the hypothesized parent distribution. All three plots show marked improvement in conformity to the parent distribution. 


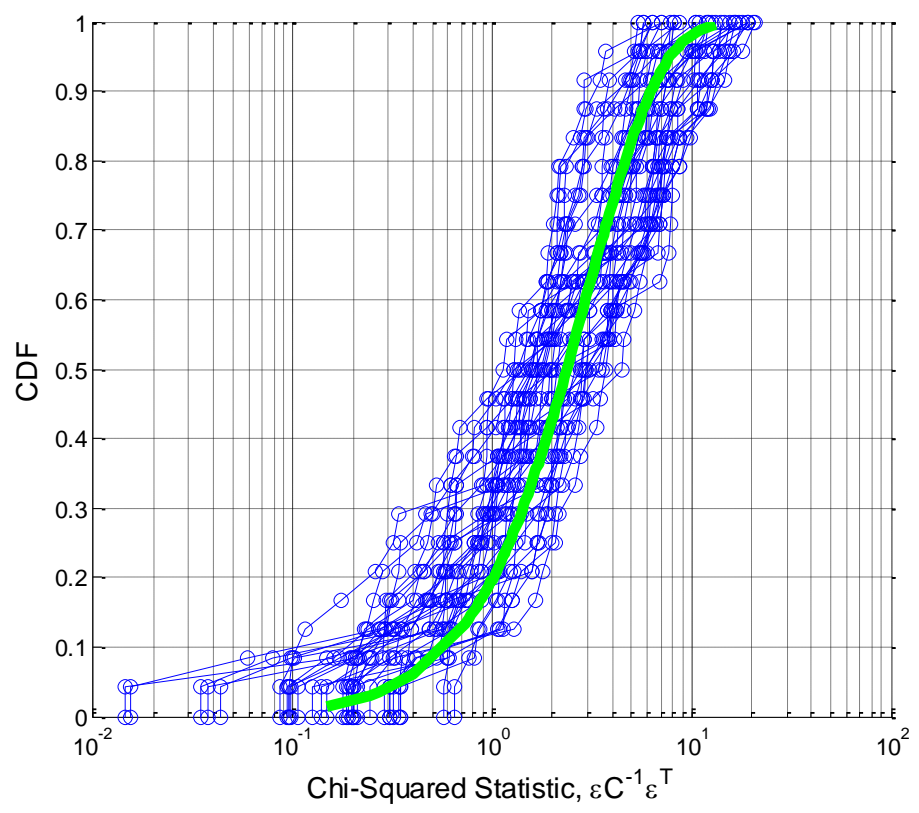

Figure 21. Empirical and hypothesized parent 3-DoF chi-squared distributions (after outlier trajectory removal and covariance tuning). The blue curves represent empirical distributions at each propagation point whereas the green curve represents the hypothesized parent distribution. The empirical distributions are conforming much closer to the hypothesized parent distribution after covariance tuning.

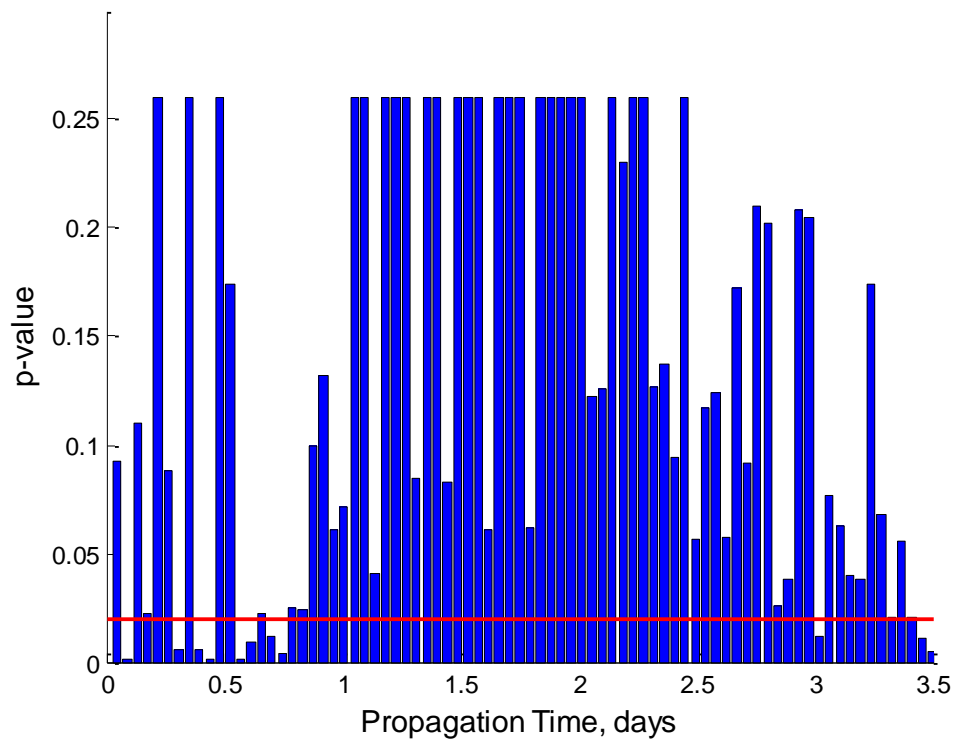

Figure 22. $p$-value vs. Propagation Time bar chart (after outlier trajectory removal and covariance tuning). The red line represents the $2 \%$ Confidence Interval whereas the blue lines each represent the likelihood the empirical distribution conforms to its parent distribution at a particular propagation point. After outlier trajectory removal and covariance tuning, $86.25 \%$ of the trajectory propagation timespan of the set of trajectories is conforming to a 3-DoF chi-squared hypothesized parent distribution.

It is likely the covariance tuning will not always achieve a Pass Percentage higher than $80 \%$ due to the aforementioned deviation between the trajectory propagation error and predicted covariance curves. Nonetheless, an Aqua and Aura covariance realism exercise across 2014 and 2015 revealed only three out of eight 3-month seasonal 
timespans that could not achieve Pass Percentages greater than $80 \%$ (with the lowest being $67.5 \%$ ). Whereas these cases are undesirable, a set of covariances exhibiting a Pass Percentage closer to $80 \%$ is a significant step towards improved $\mathrm{P}_{\mathrm{C}}$ computation. Furthermore, if it is difficult to obtain an $80 \%$ Pass Percentage over a 3.5 day propagation timespan, it is possible the RMM decision making process will lend itself to require high levels of covariance realism in a specific portion of the propagated timespan, such as between 1 and 3 days into a propagation, for which the decision to execute a maneuver is generally made at the RMM Command Authorization Meeting (CAM). Therefore, the analyst may elect to aim for a Pass Percentage that produces high levels of covariance realism between 1 and 3 days into a trajectory propagation timespan instead of the entire trajectory timespan.

\section{Automated Covariance Realism Assessment}

The EOS FDS team has developed an automated covariance realism assessment algorithm that uses a rolling 90day timespan to check the Pass Percentages of three 30-trajectory propagation sets extracted from that timespan. Each set successively begins on the first three days of the 90-day timespan and then move forward in a 3-day cadence. To be more explicit, the first dataset consists of trajectories formed on days 1, 4, 7, 10, etc., the second on days $2,5,8,11$, etc., and the third on days 3, 6, 9, 12, etc. Figure 23 below plots the three sets of mean RMS component propagation errors (with maneuvers filtered out when applicable).

The purpose of these plots is to aid the analyst in determining how to compensate for a failed assessment set should it occur amongst the three sets. The EOS FDS analyst tunes the radial and in-track covariances to the largest of the three mean RMS propagation error curves in Figure 23 at the final trajectory propagation point. If the covariance is sized to either of the smaller curves (even if either of them represent the failed assessment set), the largest curve's set will never obtain the required Pass Percentage. This strategy does not apply to the cross-track covariance since its sizing is relative to the mid-propagation point - an approach that is explained in detail in the previous section. 


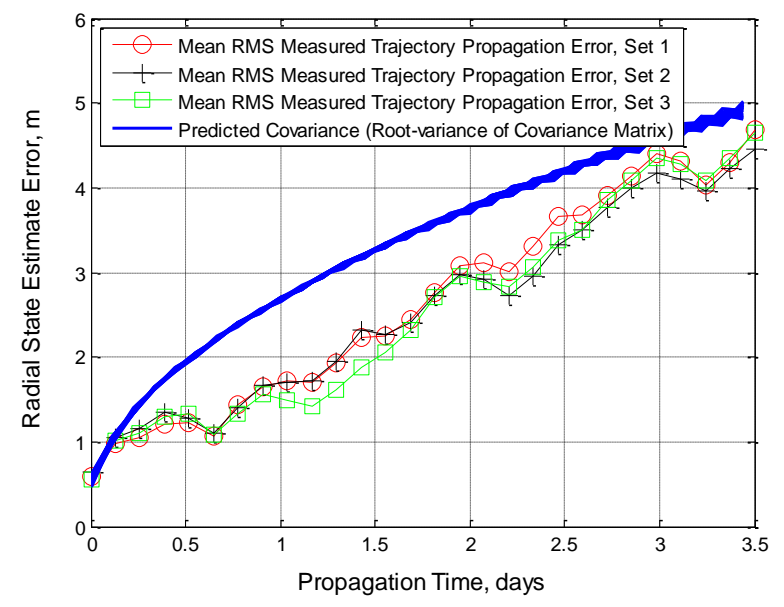

(a)

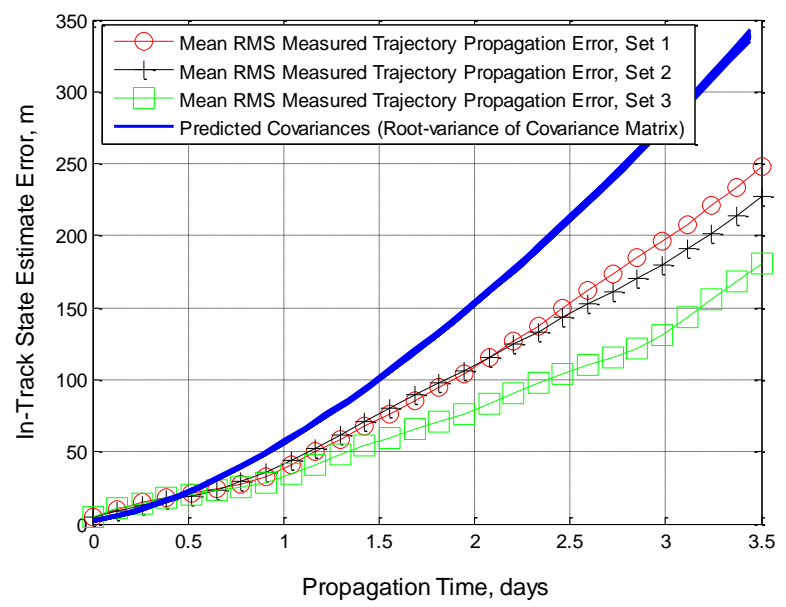

(b)

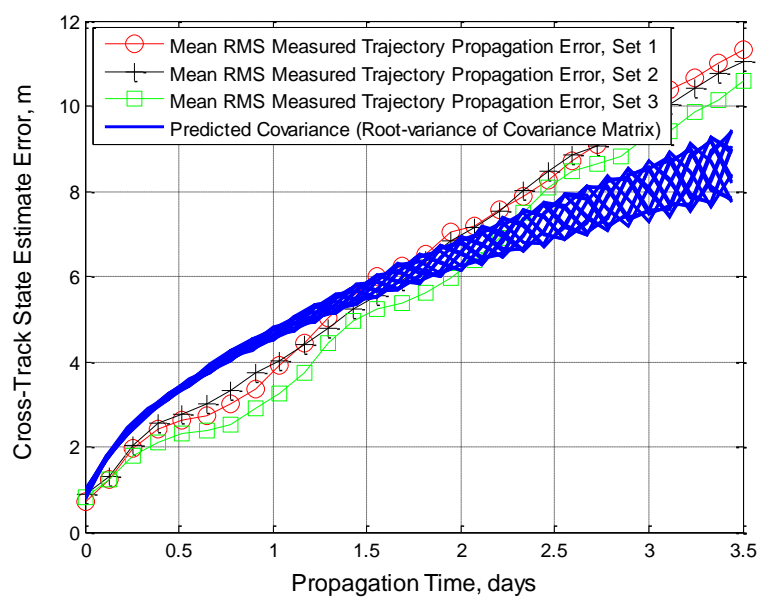

(c)

Figure 23. Component covariances (equivalent to the root-variances of the covariance matrix) vs. all three sets of mean component RMS propagation error curves. The radial and in-track covariances are tuned to their largest mean RMS propagation error curves whereas the cross-track covariance is tuned to the midpoint of its mean RMS propagation error curve.

Consequently, when the covariance realism automation determines tuning of the covariance is required, the analyst will use the steps described in the Appendix to create a new set of covariances over the 90-day timespan prior to the failed assessment date. The automation will then combine covariances from the new set of tuned trajectories and the daily covariances (being produced with the updated acceleration variances) to perform the post tuning assessment. In fact, the automation is able to selectively extract covariances using a tuning "pivot date," one that notifies the software when the last tuning of the covariance occurred. For example, a covariance assessment 10 days after a tuning date will use 81 tuned covariances from the tuning session and nine daily product covariances generated after the tuning session for the 90 -day covariance realism assessment timespan. The pivot date is reset any time a new tuning session is performed.

\section{Conclusion}

This paper demonstrates a covariance realism assessment and compensation method for the NASA EOS missions. The assessment method uses a smoothed ODTK definitive orbit and the linearized covariance propagation trajectories produced in FreeFlyer to determine the disparity between the 3-DoF chi-squared empirical distribution (of the Mahalanobis distances of the predicted trajectories) and a 3-DoF chi-squared hypothesized parent distribution. The compensation method uses SNC to inject process noise into the predicted covariances to account 
for uncertainty in the predicted force modeling dynamics. After careful trajectory outlier identification, this paper shows the covariances for the EOS missions can be successfully tuned to periods devoid of persistently high and extreme solar cavity. Finally, this paper demonstrates an automatic procedure to assess the quality of the predicted covariances and notify the operator when tuning is required.

\section{Future Work}

In the future, the EOS FDS team will not only automate the assessment of the covariances but has set up the seeds to automate the tuning of the $\mathrm{O} / \mathrm{O}$ Aqua and Aura covariances during moderate solar activity periods. A tolerance will need to be set for the differences between the radial and in-track covariances and the corresponding trajectory propagation error at the final propagation point, as well as the difference between the cross-track covariance and the corresponding trajectory propagation error at the mid propagation point. Furthermore, the EOS FDS team envisions working with the GSFC Space Weather group to determine when an outlier trajectory propagation is imminent with respect to solar activity. In such a scenario, instead of eliminating outliers, the outliers could be compensated for by the application of a $C_{D}$ consider parameter. Finally, the EOS FDS team is also investigating methods to adopt covariance realism assessment and compensation to trajectories that include maneuvers.

\section{Appendix}

This section contains the covariance realism assessment and compensation procedure use to tuned the Aqua 2014 covariance realism case study described in Section V.

Step 1: Parse the definitive trajectory overlapping the tuning timespan and store its epoch and state estimate state vector at each propagation point. The EOS FDS team uses the Consultative Committee for Space Data Systems (CCSDS) Orbit Ephemeris Message (OEM) format for this file. This format contains both the definitive state estimate and covariance of a spacecraft in the EME2000 reference frame, propagated at a 60 -second time step. ODTK provides this file format.

Step 2: Parse each predictive trajectory and store its epoch, EME2000 state vector, and EME2000 covariance matrix at each propagation point. The EOS FDS team uses the CCSDS OEM format for this file as well. FreeFlyer also provides this file format.

Step 3: Compute and store the component trajectory propagation errors, $\varepsilon$, at each predictive propagation point via the following equation:

$$
\varepsilon(t)=\left[\begin{array}{c}
x_{\text {PREDICTIVE }}-x_{\text {DEFINITIVE }} \\
y_{\text {PREDICTIVE }}-y_{\text {DEFINITIVE }} \\
z_{\text {PREDICTIVE }}-z_{\text {DEFINITIVE }}
\end{array}\right]
$$

Step 4: Rotate the predictive covariance matrices, $P(t)$, and component propagation error vectors, $\varepsilon$, from the EME2000 reference frame to the RIC, component reference frame at each predictive propagation point.

Step 5: Compute the 3-DoF chi-squared statistic for each trajectory at each propagation point using the square of the Mahalanobis distance $\left(\varepsilon P^{-1} \varepsilon^{T}\right)$ in Eq. (5).

Step 6: Compute the RMS of each component propagation error every 3 hours using the following equation:

$$
\varepsilon_{\mathrm{RMS}}=\sqrt{\varepsilon_{\text {mean }}^{2}+\varepsilon_{\text {standard deviation }}^{2}}
$$

Step 7: Standardize each component trajectory propagation error by its corresponding predictive covariance, $\sigma_{i}$, at each common propagation point using the following equation: 


$$
\left|\varepsilon_{\mathrm{R}}\right|=\frac{\varepsilon_{\mathrm{R}}}{\sigma_{\mathrm{R}}},\left|\varepsilon_{\mathrm{I}}\right|=\frac{\varepsilon_{\mathrm{I}}}{\sigma_{\mathrm{I}}},\left|\varepsilon_{\mathrm{C}}\right|=\frac{\varepsilon_{\mathrm{C}}}{\sigma_{\mathrm{C}}}
$$

This standardization will transform the dataset to standardized normal variables (z-variables).

Step 8: Determine the hypothesized parent and empirical distributions for the 3-DoF chi-squared statistics computed in Step 5 and the standard component errors in Step 7. Note the hypothesized parent distributions can be calculated once and used in perpetuity for covariance tuning processes in the future.

Step 9: Determine the $p$-value for 3-DoF chi-squared EDF test statistic against its parent distribution at each predictive trajectory propagation point and store the results into an array.

Step 10: Normalize the final group of standard in-track propagation errors at the final propagation point as follows:

$$
\underset{\text { In-Track Error }}{\text { Normalized Standard }}=\frac{\left|\varepsilon_{\mathrm{I}}\right|-\text { mean }\left|\varepsilon_{\mathrm{I}}\right|}{\operatorname{stddev}\left|\varepsilon_{\mathrm{I}}\right|}
$$

Step 11: Store all values that are outside $1 \sigma$ from the group of standard in-track errors (at the final propagation point) into an array in order from most to least deviant.

Step 12: Run the Rosner Outlier Identification test to determine if any of the deviates in the preceding step are outliers; if so remove the trajectories corresponding to the outliers from the analysis. The potential outlier trajectories must be entered into the test from most to least deviant. A confidence level must be set that acts as a threshold over which a trajectory is not an outlier. For this analysis a $2 \%$ confidence interval is used to identify outliers. Additionally, potential trajectory outliers are tested together in groups. For example, the first potential outlier is tested against the entire dataset, then the first and second potential outliers are tested, then the first, second and third potential outliers are tested. The resulting $p$-values correspond to each test group instead of each specific potential outlier. Meaning, the first $p$-value corresponds to the first potential outlier but the second $p$-value corresponds to the first and second potential outliers. Therefore, if the second $p$-value is less than the confidence interval, both potential outliers must be removed.

Step 13: Adjust the in-track and radial acceleration variances set in Eq. (7), leave the $C_{D}$ variance fixed, and repropagate a single trajectory until its final radial and in-track covariance points are above the corresponding final mean RMS propagation error data points. In the same propagation run, adjust the cross-track acceleration variance set in Eq. (7) until its mid-propagation covariance point intersects the corresponding mean RMS propagation error data point.

\section{Acknowledgments}

The authors are grateful for the technical review of the covariance realism assessment and compensation method demonstrated in this paper by Dr. Russell Carpenter (Senior Aerospace Engineer, NASA Goddard Space Flight Center) and Dr. Anne Long (Chief System Engineer, a.i. solutions, Inc.). The authors would also like to thank the software testing and development by the entire EOS FDS team. W. H. Zaidi specifically thanks EOS Orbit Analysts Brian McCarthy and Juan Ojeda-Romero for their expertise in MATLAB, EOS Orbit Analyst Ryan Moore for his expertise in FreeFlyer and software automation, EOS Orbit Analyst Shawn Hoffman for his expertise in FreeFlyer, EOS Orbit Analyst Avery Ruel for her expertise in software acceptance testing, and EOS Orbit Analyst Scott Patano, Dr. Geoff Wawrzyniak (Project Engineer, a.i. solutions, Inc.), and David Tracewell (Senior Aerospace Engineer, NASA Goddard Space Flight Center) for their invaluable editorial comments applied to this paper.

\section{References}

${ }^{1}$ Xian-Zong, B., Lei, C., and Guo-Jin, T., "Explicit expression of collision probability in terms of RSW," Advances in Space Research, Vol. 52, No. 6, pp. 1078-1096.

${ }^{2}$ Hujsak, R. S., Woodburn, J. W., and Seago, J. H., "The Orbit Determination Tool Kit (ODTK) - Version 5," AAS/AIAA Spaceflight Mechanics Conference, AAS, Sedona, AZ, 2007. 
${ }^{3}$ Duncan, M., and Long, A., "Realistic Covariance Prediction for the Earth Science Constellation," AIAA/AAS Astrodynamics Specialist Conference and Exhibit, AIAA, Keystone, CO, 2006.

${ }^{4}$ Horwood, J. T., Aristoff, J. M., Singh, N., Poore, A. B., and Hejduk, M. D., "Beyond covariance realism: a new metric for uncertainty realism," SPIE Proceedings: Signal and Data Processing of Small Targets, Vol. 9092, 2014.

${ }^{5}$ Rosner, B., "On the Detection of Many Outliers," Technometrics, Vol. 17, 1975, pp. 221-227.

${ }^{6}$ Rosner, B., "Percentage Points for the RST Many Outlier Procedure," Technometrics, Vol. 19, pp. 307-312.

${ }^{7}$ D'Agostino, R. B., and Stephens, M. A., Goodness-of-Fit Techniques (Statistics: a Series of Textbooks and Monographs, Vol. 68), $1^{\text {st }}$ ed., Marcel Dekker, New York, 1986.

${ }^{8}$ Grubbs, F. E., "Procedures for detecting Outlying Observations in Samples," Technometrics, Vol. 19, 1969, pp. 1-21. 